\title{
A Multiscale Algorithm for Heat Conduction-Radiation Problems in Porous Materials with Quasi-Periodic Structures
}

\author{
Zhiqiang Yang ${ }^{1, *}$, Yi Sun ${ }^{1}$, Junzhi Cui ${ }^{2}$ and Xiao $\mathrm{Li}^{1}$ \\ ${ }^{1}$ Department of Astronautic Science and Mechanics, Harbin Institute of Technology, \\ Harbin 150001, P.R. China. \\ ${ }^{2}$ LSEC, ICMSEC, Academy of Mathematics and Systems Sciences, Chinese Academy \\ of Sciences, Beijing 100190, P.R. China.
}

Received 8 May 2017; Accepted (in revised version) 31 July 2017

\begin{abstract}
This paper develops a second-order multiscale asymptotic analysis and numerical algorithms for predicting heat transfer performance of porous materials with quasi-periodic structures. In these porous materials, they have periodic configurations and associated coefficients are dependent on the macro-location. Also, radiation effect at microscale has an important influence on the macroscopic temperature fields, which is our particular interest in this study. The characteristic of the coupled multiscale model between macroscopic scale and microscopic scale owing to quasi-periodic structures is given at first. Then, the second-order multiscale formulas for solving temperature fields of the nonlinear problems are constructed, and associated explicit convergence rates are obtained on some regularity hypothesis. Finally, the corresponding finite element algorithms based on multiscale methods are brought forward and some numerical results are given in detail. Numerical examples including different coefficients are given to illustrate the efficiency and stability of the computational strategy. They show that the expansions to the second terms are necessary to obtain the thermal behavior precisely, and the local and global oscillations of the temperature fields are dependent on the microscopic and macroscopic part of the coefficients respectively.
\end{abstract}

AMS subject classifications: 35B27, 34E13, 74Q05, 83C30

Key words: Multiscale asymptotic analysis, radiation boundary condition, quasi-periodic structures, nonlinear heat transfer problems.

\section{Introduction}

Porous materials are widely applied in the aeronautic and aerospace engineering owing to the good thermal stability, low relative density and high heat resistance. In particu-

*Corresponding author. Email addresses: yangzhiqiang@hit.edu.cn (Z. Yang), sunyi@hit.edu.cn (Y. Sun), cjz@lsec.cc.ac.cn (J. Cui), xli_90@163.com (X. Li) 
lar, porous materials are usually designed for thermal protection system (TPS) during the spacecraft's flying out or re-entry into the atmosphere. Under such condition, the temperature of spacecraft's surface will be high enough in a moment, and heat radiation should not be omitted in actual calculations. Also, as the materials often have periodic configurations and characteristic coefficients oscillate sharply in small cells, it is necessary and important to develop an effective method to predict the thermal and mechanical performance of the porous materials.

Solving the heat radiative problem in porous materials by a direct numerical method becomes rather difficult since it would cost huge computer memories and time to accurately catch the local fluctuation behavior of temperature fields even for the supercomputer. Generally, homogenization methods and associated multiscale algorithms describe the global behavior by reduce the governing equations with rapidly varying coefficients to the equations with effective coefficients, which can not only save the computing resources but also guarantee the calculation precision [1-7]. Moreover, by adding appropriate correctors, the approximate solutions with oscillatory behavior can also be reproduced availably [8-10]. Up to now, some homogenization and multiscale methods were developed to study the heat conduction-radiation problems arising from porous materials. Liu and Zhang [11] investigated the effective macroscopic properties of radiativeconductive heat transfer problems in periodic porous materials. Bakhvalov [12] gave the asymptotic expansion forms for the solutions of those problems. Allaire and El Ganaoui [13] studied the heat transfer problems with $\varepsilon^{-1}$-order radiation boundary by two-scale expansion methods, and justified the convergence. Meanwhile, Ma and Cui [14] proposed a second-order two-scale method to solve the coupled problems, and obtained the convergence order with $\mathcal{O}\left(\varepsilon^{1 / 2}\right)$. Cui et al. [15-17] discussed the heat conduction and radiation problems in periodic or random porous materials, and developed a newly higher-order multiscale method for the problems. Later, Yang, Sun and Cui $[18,19]$ obtained the second-order multiscale solutions for the dynamic thermo-elastic problems of porous materials with interior surface radiation. Obviously, from the works mentioned previously, the homogenization method and the associated multiscale techniques can give sufficiently effective predictions of the thermal and thermo-mechanical coupling properties of arbitrarily sophisticated microstructures. Also, such techniques and algorithms can perform calculation of the temperature and heat flux fields on the macro scale according to the effective coefficients obtained at the microscopic scale.

Generally speaking, owing to composite materials manufacturing technology, such as fatigue damages, the material parameters are no longer periodic, but local-periodic, i.e., quasi-periodic. In other words, the material coefficients can depend not only on the microscale information but also on the macro location. Functionally gradient materials (FGM) are a representative material with quasi-periodic structures [20-23]. Based on the traditional homogenization methods, Lions [1] systematically investigated the elliptical boundary value problems with quasi-periodic structures. Wirth [24] proposed a space dimensions method multiplied by different scales to efficiently treat quasi-periodic and multiscale problems. Andrianov et al. [25] applied a novel asymptotical approach to in- 
tegrate differential equations arising from quasi-regular structures. Cao [26] gave a firstorder multiscale expansion and detailed theoretical analysis for linear elasticity problems with quasi-periodic structures. Further, Ma and Su $[9,27,28]$ developed a secondorder multiscale method for heat conduction problems, linear elasticity and thermoelastic problems with quasi-periodic structures. Recently, Don et al. [29] performed a second-order two-scale analysis method for the damped wave equations of composite materials with a quasi-periodic structure, and obtained the error estimates based on some reasonable assumptions. However, the previous multiscale asymptotic expansions and algorithms cannot be directly employed to the heat conduction-radiation problems due to the nonlinearity of the coupled problems and the complexity microstructure of porous materials.

In this work, we will mainly discuss the heat conduction-radiation problems of porous materials with a quasi-periodic structure. Particularly, the nonlinear heat radiation account for the scale effect and sophisticated microstructures of the porous materials are considered in this study. The radiation boundary condition was investigated by Liu and Zhang [11], Bakhvalov [12] and Yang et al. [15-19], and it is a classical physical model in practical applications. This paper is to establish a novel high-order multiscale method with less effort and computational cost to give a better approximation. We introduce correction terms into the first-order asymptotic expansions of the temperature fields, define a series of cell functions, and then obtain the approximate error estimates under some regularity hypothesis.

The outline of this paper is organized as follows. Section 2 describes the detailed governing equations of heat conduction-radiation problems of porous materials with quasiperiodic structures. In Section 3, the multiscale asymptotic expansions for the nonlinear heat transfer problems are presented. In Section 4, the error estimates on the approximate solutions are analyzed under some reasonable assumptions, and the multiscale finite element algorithms are given in Section 5. In Section 6, some numerical results are demonstrated to verify the validity of the multiscale algorithms. Finally, some conclusions are presented in Section 7.

Throughout the paper the Einstein summation convention on repeated indices is adopted.

\section{Governing equations of heat conduction-radiation problems}

In this section, the heat conduction-radiation equations in porous materials with quasiperiodic structures are constructed in detail.

Let $Y=\left\{y: 0 \leq y_{j} \leq 1, j=1,2,3\right\}$ and $\omega$ be an unbounded domain of $R^{3}$ which satisfies following conditions:

(B1) $\omega$ is a smooth domain of $R^{3}$ with a periodic structure.

(B2) The unit cell of periodicity $Y^{*}=\omega \cap Y$ is a domain with a Lipschitz boundary, as illustrated in Fig. 1(b). 
(B3) The set $Y \backslash \bar{\omega}$ and the intersection of $Y \backslash \bar{\omega}$ with the $\delta_{0}\left(\delta_{0}<<1\right)$ neighborhood of $\partial Y$ consist of finite number of Lipschitz domains separated from each other and from the edges of the cube $Y$ by a positive distance.

(B4) The cavities are convex.

Then, the domain $\Omega^{\varepsilon}$ has the form: $\Omega^{\varepsilon}=\Omega \cap \varepsilon \omega$, as shown in Fig. 1(a), where $\Omega$ is a bounded Lipschitz convex domain without pores. The surfaces of cavities are supposed to be diffuse and grey, i.e., the emissivity $e$ of the surfaces does not depend on the wavelength and direction of the radiation. Here $\varepsilon>0$ is a small parameter, and it denotes the relative size between a unit cell and the whole domain of the materials.

The heat radiation problems of porous materials with rapidly oscillatory coefficients can be defined in a closed cavity as follows:

$$
-v_{i} k_{i j}\left(x, \frac{x}{\varepsilon}\right) \frac{\partial T_{\varepsilon}(x, t)}{\partial x_{j}}=e \sigma T_{\varepsilon}^{4}(x, t)-e \int_{\Gamma_{\varepsilon, m}^{c}} R_{\varepsilon}(z, t) F(x, z) d z \text {, on } \Gamma_{\varepsilon}^{c}
$$

where $T_{\varepsilon}(x, t)$ denotes the temperature fields, $\sigma$ is the Stefan-Boltzmann constant, and $\mathbf{v}=\left(v_{i}\right), i=1,2,3$ is the unit outward normal on surfaces $\Gamma_{\varepsilon}^{c}$ of the cavities. $R_{\varepsilon}(z, t)$ is the intensity of emitted radiation defined by

$$
R_{\varepsilon}(x, t)=e \sigma T_{\varepsilon}^{4}(x, t)+(1-e) \int_{\Gamma_{\varepsilon, m}^{c}} R_{\varepsilon}(z, t) F(x, z) d z, \forall x \in \Gamma_{\varepsilon, m}^{c}
$$

where $\Gamma_{\varepsilon, m}^{c}$ is the interior boundary of a cavity, such that $\Gamma_{\varepsilon}^{c}=\bigcup_{i=1}^{m(\varepsilon)} \Gamma_{\varepsilon, i^{\prime}}^{c}$ and $m(\varepsilon)$ is the number of cavities in the porous materials. $F(x, z)$ denotes the view factor, and is defined on 3-D for a convex cavity as follows (Refs. [12-15]):

$$
F(x, z)=\frac{n_{z} \cdot(x-z) n_{x} \cdot(z-x)}{\pi|z-x|^{4}}
$$

where $n_{z}$ is the unit normal at the point $z$, and for any $(x, z) \in\left(\Gamma_{\varepsilon, m}^{c}\right)^{2}$ (for a closed surface), which satisfies the following properties:

$$
F(x, z) \geq 0, \quad F(x, z)=F(z, x), \quad \int_{\Gamma_{\varepsilon, m}^{c}} F(x, z) d z=1
$$

Under the aforementioned assumptions, we consider the heat conduction-radiation 


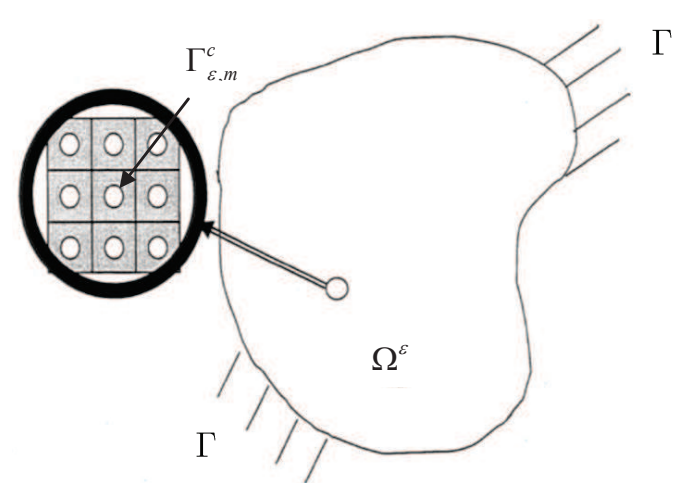

(a)

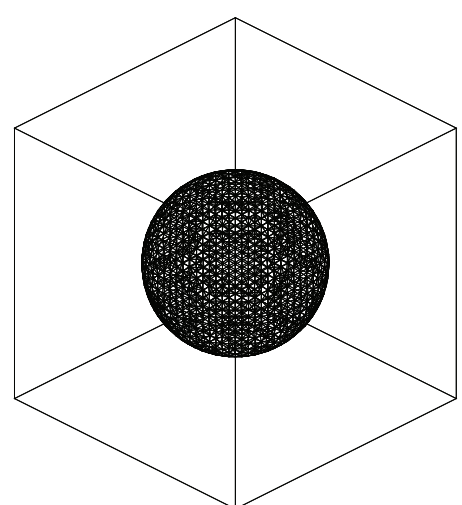

(b)

Figure 1: Periodic distribution of porous materials: (a) Domain $\Omega^{\varepsilon}$; (b) Unit cell $Y^{*}$.

equations with initial-boundary value conditions for a given structure as follows:

$$
\left\{\begin{array}{l}
\frac{\partial T_{\varepsilon}(x, t)}{\partial t}-\frac{\partial}{\partial x_{i}}\left(k_{i j}\left(x, \frac{x}{\varepsilon}\right) \frac{\partial T_{\varepsilon}(x, t)}{\partial x_{j}}\right)=f(x, t), \quad(x, t) \in \Omega^{\varepsilon} \times\left(0, t_{*}\right), \\
T_{\varepsilon}(x, t)=\bar{T}, \quad(x, t) \in \Gamma \times\left(0, t_{*}\right) \\
T_{\mathcal{E}}(x, 0)=T_{i n}(x), \quad x \in \Omega^{\varepsilon}, \\
-v_{i} k_{i j}\left(x, \frac{x}{\varepsilon}\right) \frac{\partial T_{\varepsilon}(x, t)}{\partial x_{j}} \\
\quad=e \sigma T_{\varepsilon}^{4}(x, t)-e \int_{\Gamma_{\varepsilon, m}^{c}} R_{\varepsilon}(z, t) F(x, z) d z, \quad(x, t) \in \Gamma_{\varepsilon}^{c} \times\left(0, t_{*}\right)
\end{array}\right.
$$

where $f(x, t)$ is the internal thermal source; $T_{\text {in }}(x)$ denotes the initial temperature fields. The boundary can be expressed as $\partial \Omega^{\varepsilon}=\Gamma \cup \Gamma_{\varepsilon}^{c}$, $\Gamma$ denotes the external boundary portions where temperature fields are prescribed. $\bar{T}$ is the value of a temperature component. $k_{i j}\left(x, \frac{x}{\varepsilon}\right)(i, j=1,2,3)$ is the thermal conductivity tensor, and satisfies the conditions of symmetry

$$
k_{i j}\left(x, \frac{x}{\varepsilon}\right)=k_{j i}\left(x, \frac{x}{\varepsilon}\right) \text {, }
$$

and ellipticity

$$
\lambda_{1} \gamma_{i} \gamma_{i} \leqslant k_{i j}\left(x, \frac{x}{\varepsilon}\right) \gamma_{i} \gamma_{j} \leqslant \lambda_{2} \gamma_{i} \gamma_{i}
$$

where $\lambda_{1}$ and $\lambda_{2}$ are constants independent of $x$, and $\gamma_{i}(i=1,2,3)$ is an arbitrary vector with real elements. Let $y=x / \varepsilon, y$ denotes the local coordinate, then temperature fields $T_{\varepsilon}(x, t)$ and heat conduction coefficients $k_{i j}\left(x, \frac{x}{\varepsilon}\right)(i, j=1,2,3)$ change into $T(x, y, t)$ and $k_{i j}(x, y)$, respectively. Since the physical parameters of the porous materials are no longer periodic, but local-periodic, i.e., quasi-periodic, the conduction coefficients depend not only on the microscale but also on the specific location of the macroscale. For simplicity, let $T_{\varepsilon}=T_{\varepsilon}(x, t), T_{0}=T_{0}(x, t)$ and $f=f(x, t)$ in the following. 
Remark 2.1. By suppositions (B1)-(B4), the existence and uniqueness of the weak solution $T_{\varepsilon}(x, t)$ for the initial-boundary value problems (2.3) can be established (see Refs. [30,31]).

\section{The multiscale asymptotic expansions}

In this section, the multiscale asymptotic expansion methods are developed to solve the transient heat conduction-radiation problems (2.3) of porous materials with quasiperiodic structures.

\subsection{Cell problems, homogenized problems and homogenized coefficients}

Firstly, enlightened by Refs. [1,2], $T_{\mathcal{E}}(x, t)$ can be expanded into a series in the following form:

$$
T_{\varepsilon}(x, t)=T_{0}(x, y, t)+\varepsilon T_{1}(x, y, t)+\varepsilon^{2} T_{2}(x, y, t)+\cdots,
$$

Then

$$
\begin{aligned}
T_{\varepsilon}^{4}(x, t) & =\left(T_{0}(x, y, t)+\varepsilon T_{1}(x, y, t)+\varepsilon^{2} T_{2}(x, y, t)+\cdots\right)^{4} \\
& =T_{0}^{4}+\varepsilon\left(4 T_{0}^{3} T_{1}\right)+\varepsilon^{2}\left(6 T_{0}^{2} T_{1}^{2}+4 T_{0}^{3} T_{2}\right)+\cdots .
\end{aligned}
$$

Owing to $y=x / \varepsilon$ for $y \in Y$, the partial derivative can be defined as:

$$
\frac{\partial}{\partial x_{i}} \rightarrow \frac{\partial}{\partial x_{i}}+\frac{1}{\varepsilon} \frac{\partial}{\partial y_{i}}, \quad i=1,2,3
$$

Substituting (3.1) and (3.3) into (2.3) and considering the coefficients of $\varepsilon^{-2}, \varepsilon^{-1}, \varepsilon^{0}$, one obtains the following three equations:

$$
\begin{gathered}
\mathcal{O}\left(\varepsilon^{-2}\right):-\frac{\partial}{\partial y_{i}}\left(k_{i j}(x, y) \frac{\partial T_{0}}{\partial y_{j}}\right)=0 \\
\mathcal{O}\left(\varepsilon^{-1}\right):-\frac{\partial}{\partial y_{i}}\left(k_{i j}(x, y) \frac{\partial T_{1}}{\partial y_{j}}\right)-\frac{\partial}{\partial y_{i}}\left(k_{i j}(x, y) \frac{\partial T_{0}}{\partial x_{j}}\right)-\frac{\partial}{\partial x_{i}}\left(k_{i j}(x, y) \frac{\partial T_{0}}{\partial y_{j}}\right)=0 \\
\mathcal{O}\left(\varepsilon^{0}\right):-\frac{\partial}{\partial y_{i}}\left(k_{i j}(x, y) \frac{\partial T_{2}}{\partial y_{j}}\right)-\frac{\partial}{\partial y_{i}}\left(k_{i j}(x, y) \frac{\partial T_{1}}{\partial x_{j}}\right)-\frac{\partial}{\partial x_{i}}\left(k_{i j}(x, y) \frac{\partial T_{1}}{\partial y_{j}}\right) \\
-\frac{\partial}{\partial x_{i}}\left(k_{i j}(x, y) \frac{\partial T_{0}}{\partial x_{j}}\right)=f(x, t) .
\end{gathered}
$$

Also, considering the radiative boundary terms given in (2.3), we have that

$$
-v_{i} k_{i j}(x, y)\left(\frac{\partial}{\partial x_{i}}+\frac{1}{\varepsilon} \frac{\partial}{\partial y_{i}}\right)=-v_{i} \frac{1}{\varepsilon} k_{i j}(x, y) \frac{\partial}{\partial y_{i}}-v_{i} k_{i j}(x, y) \frac{\partial}{\partial x_{i}} \text {. }
$$

Let

$$
B_{\varepsilon}=\varepsilon^{-1} B_{0}+B_{1}
$$


where

$$
\begin{aligned}
& B_{0}=-v_{i} k_{i j}(y) \frac{\partial}{\partial y_{i}}, \\
& B_{1}=-v_{i} k_{i j}(y) \frac{\partial}{\partial x_{i}} .
\end{aligned}
$$

Similarly to (3.1), $R_{\varepsilon}(x, t)$ has the following multiscale asymptotic expansions

$$
R_{\varepsilon}(x, t)=R_{0}(x, t)+\varepsilon R_{1}(x, y, t)+\varepsilon^{2} R_{2}(x, y, t)+\cdots .
$$

Taking (3.2) into the radiation terms of (2.3) and utilizing (3.8)-(3.10) can lead to the following identities

$$
\begin{aligned}
B_{\varepsilon} T_{\varepsilon}= & \varepsilon^{-1} B_{0} T_{0}+B_{1} T_{0}+B_{0} T_{1}+\varepsilon\left(B_{1} T_{1}+B_{0} T_{2}\right) \\
= & e \sigma T_{\varepsilon}^{4}(x, t)-e \int_{\Gamma_{\varepsilon, i}^{c}} R_{\varepsilon}(z, t) F(x, z) d z \\
= & e \sigma T_{0}^{4}(x, y, t)-e \int_{\Gamma^{c}} R_{0}(x, t) F(y, s) d s \\
& +\varepsilon\left(4 e \sigma T_{0}^{3}(x, y, t) T_{1}(x, y, t)-e \int_{\Gamma^{c}} R_{1}(x, s, t) F(y, s) d s\right) \\
& +\mathcal{O}\left(\varepsilon^{2}\right), \\
R_{0}(x, t) & +\varepsilon R_{1}(x, y, t)+\varepsilon^{2} R_{2}(x, y, t) \\
= & e \sigma T_{0}^{4}(x, y, t)+(1-e) \int_{\Gamma^{c}} R_{0}(x, t) F(y, s) d s \\
& +\varepsilon\left(4 e \sigma T_{0}^{3}(x, y, t) T_{1}(x, y, t)+(1-e) \int_{\Gamma^{c}} R_{1}(x, s, t) F(y, s) d s\right) \\
& +\mathcal{O}\left(\varepsilon^{2}\right),
\end{aligned}
$$

where $s=z / \varepsilon, z$ denotes the macroscopic coordinate, and $s$ the local coordinate.

From (3.12) and equating the coefficients of the same powers for $\varepsilon$, two equalities can be obtained as follows:

$$
\begin{aligned}
& R_{0}(x, t)=\sigma T_{0}^{4}(x, y, t), \\
& R_{1}(x, y, t)=4 e \sigma T_{0}^{3}(x, y, t) T_{1}(x, y, t)+(1-e) \int_{\Gamma^{c}} R_{1}(x, s, t) F(y, s) d s .
\end{aligned}
$$

Therefore, taking into account (3.4)-(3.6) and (3.11)-(3.14), following boundary value problems are given by:

$$
\left\{\begin{array}{l}
-\frac{\partial}{\partial y_{i}}\left(k_{i j}(x, y) \frac{\partial T_{0}(x, y, t)}{\partial y_{j}}\right)=0, \text { in } Y^{*}, \\
-v_{i} k_{i j}(x, y) \frac{\partial T_{0}(x, y, t)}{\partial y_{j}}=0, \quad y \in \Gamma^{c},
\end{array}\right.
$$




$$
\begin{aligned}
& \left(-\frac{\partial}{\partial y_{i}}\left(k_{i j}(x, y) \frac{\partial T_{1}(x, y, t)}{\partial y_{j}}\right)=\frac{\partial}{\partial y_{i}}\left(k_{i j}(x, y) \frac{\partial T_{0}(x, y, t)}{\partial x_{j}}\right)\right. \\
& +\frac{\partial}{\partial x_{i}}\left(k_{i j}(x, y) \frac{\partial T_{0}(x, y, t)}{\partial y_{j}}\right), \quad \text { in } Y^{*}, \\
& -v_{i} k_{i j}(x, y)\left(\frac{\partial T_{1}(x, y, t)}{\partial y_{j}}+\frac{\partial T_{0}(x, y, t)}{\partial x_{j}}\right) \\
& =e \sigma T_{0}^{4}(x, y, t)-e \int_{\Gamma^{c}} R_{0}(x, t) F(y, s) d s, \quad y \in \Gamma^{c}, \\
& \left(\frac{\partial T_{0}(x, y, t)}{\partial t}-\frac{\partial}{\partial y_{i}}\left(k_{i j}(x, y) \frac{\partial T_{2}(x, y, t)}{\partial y_{j}}\right)=f(x, t)+\frac{\partial}{\partial y_{i}}\left(k_{i j}(x, y) \frac{\partial T_{1}(x, y, t)}{\partial x_{j}}\right)\right. \\
& +\frac{\partial}{\partial x_{i}}\left(k_{i j}(x, y) \frac{\partial T_{0}(x, y, t)}{\partial x_{j}}\right)+\frac{\partial}{\partial x_{i}}\left(k_{i j}(x, y) \frac{\partial T_{1}(x, y, t)}{\partial y_{j}}\right), \quad \text { in } Y^{*}, \\
& -v_{i}\left(k_{i j}(x, y) \frac{\partial T_{2}(x, y, t)}{\partial y_{j}}+k_{i j}(x, y) \frac{\partial T_{1}(x, y, t)}{\partial x_{j}}\right) \\
& =4 e \sigma T_{0}^{3}(x, y, t) T_{1}(x, y, t)-e \int_{\Gamma^{c}} R_{1}(x, s, t) F(y, s) d s, \quad y \in \Gamma^{c},
\end{aligned}
$$

where $\Gamma^{c}$ is the surface boundary of the cavities in $Y^{*}$, and $Y^{*}$ is the solid part of $Y$.

It can be easily found from (3.13) and (3.15) that $T_{0}(x, y, t)$ is only dependent on macroscopic variable $x$.

Thus, after some simplification, (3.16) can be rewritten as

$$
\left\{\begin{array}{l}
-\frac{\partial}{\partial y_{i}}\left(k_{i j}(x, y) \frac{\partial T_{1}(x, y, t)}{\partial y_{j}}\right)=\frac{\partial}{\partial y_{i}}\left(k_{i j}(x, y) \frac{\partial T_{0}(x, t)}{\partial x_{j}}\right), \text { in } Y^{*}, \\
-v_{i} k_{i j}(x, y)\left(\frac{\partial T_{1}(x, y, t)}{\partial y_{j}}+\frac{\partial T_{0}(x, t)}{\partial x_{j}}\right) \\
\quad=e \sigma T_{0}^{4}(x, t)-e \int_{\Gamma^{c}} R_{0}(x, t) F(y, s) d s, \quad y \in \Gamma^{c} .
\end{array}\right.
$$

Further, owing to $\int_{\Gamma^{c}} F(y, s) d s=1$, it is easy to get that

$$
e \sigma T_{0}^{4}(x, t)-e \int_{\Gamma^{c}} R_{0}(x, t) F(y, s) d s=0
$$

Then, the solution of (3.18) can be defined the following form

$$
T_{1}(x, y, t)=N_{\alpha_{1}}(x, y) \frac{\partial T_{0}}{\partial x_{\alpha_{1}}}, \quad \alpha_{1}=1,2,3
$$


Combining (3.18)-(3.20), the auxiliary functions $N_{\alpha_{1}}(x, y)\left(\alpha_{1}=1,2,3\right)$ defined on 1normalized cell $Y^{*}$ satisfy the problems given by

$$
\left\{\begin{array}{l}
\frac{\partial}{\partial y_{i}}\left(k_{i j}(x, y) \frac{\partial N_{\alpha_{1}}(x, y)}{\partial y_{j}}\right)=-\frac{\partial k_{i \alpha_{1}}(x, y)}{\partial y_{i}}, \text { in } Y^{*}, \\
-v_{i}\left(k_{i j}(x, y) \frac{\partial N_{\alpha_{1}}(x, y)}{\partial y_{j}}+k_{i \alpha_{1}}(x, y)\right)=0, \quad y \in \Gamma^{c}, \\
N_{\alpha_{1}}(x, y) \text { is } Y \text {-periodic. }
\end{array}\right.
$$

Similarly to Lemma 2.2 in Ref. [16], it is proved that problems (3.21) have a unique solution.

By analogy, by virtue of (3.11) and (3.20), (3.17) can be rewritten as

$$
\left\{\begin{array}{c}
\frac{\partial T_{0}(x, t)}{\partial t}-\frac{\partial}{\partial y_{i}}\left(k_{i j}(x, y) \frac{\partial T_{2}(x, y, t)}{\partial y_{j}}\right)=\frac{\partial}{\partial y_{i}}\left(k_{i j}(x, y) N_{\alpha_{1}}(x, y)\right) \frac{\partial^{2} T_{0}}{\partial x_{j} \partial x_{\alpha_{1}}} \\
\quad+k_{i j}(x, y) \frac{\partial^{2} T_{0}}{\partial x_{i} \partial x_{j}}+k_{i j}(x, y) \frac{\partial N_{\alpha_{1}}(x, y)}{\partial y_{j}} \frac{\partial^{2} T_{0}}{\partial x_{i} \partial x_{\alpha_{1}}}+f(x, t), \text { in } Y^{*}, \\
-v_{i}\left(k_{i j}(x, y) \frac{\partial T_{2}(x, y, t)}{\partial y_{j}}+k_{i j}(x, y) N_{\alpha_{1}}(x, y) \frac{\partial^{2} T_{0}}{\partial x_{j} \partial x_{\alpha_{1}}}\right) \\
=4 e \sigma T_{0}^{3}(x, t) N_{\alpha_{1}}(x, y) \frac{\partial T_{0}}{\partial x_{\alpha_{1}}}-e \int_{\Gamma^{c}} R_{1}(x, s, t) F(y, s) d s, \quad y \in \Gamma^{c} .
\end{array}\right.
$$

Also, taking into account equality (3.14), we have

$$
R_{1}(x, y, t)=4 e \sigma T_{0}^{3}(x, y, t) T_{1}(x, y, t)+(1-e) \int_{\Gamma^{c}} R_{1}(x, s, t) F(y, s) d s .
$$

After integrating on both sides of the equation with respect to $y$, (3.23) can be rewritten as

$$
\int_{\Gamma^{c}} R_{1}(x, y, t) d y=\int_{\Gamma^{c}} 4 e \sigma T_{0}^{3}(x, t) T_{1}(x, y, t) d y+(1-e) \int_{\Gamma^{c}} \int_{\Gamma^{c}} R_{1}(x, s, t) F(y, s) d s d y .
$$

Then, taking the property $\int_{\Gamma^{c}} F(s, y) d y=1$ yields the equality

$$
\int_{\Gamma^{c}} 4 \sigma T_{0}^{3}(x, t) T_{1}(x, y, t) d y=\int_{\Gamma^{c}} R_{1}(x, y, t) d y .
$$

Thus, we obtain the following results

$$
\int_{\Gamma^{c}}\left(4 e \sigma T_{0}^{3}(x, t) T_{1}(x, y, t)-e \int_{\Gamma^{c}} R_{1}(x, s, t) F(y, s) d s\right) d y=0 .
$$


By an integral average over $Y$ and combining (3.22)-(3.26), the homogenized equations associated with (2.3) are obtained as:

$$
\left\{\begin{array}{rc}
\frac{\left|Y^{*}\right|}{|Y|} \frac{\partial T_{0}(x, t)}{\partial t}-\frac{\partial}{\partial x_{i}}\left(\hat{k}_{i j}(x) \frac{\partial T_{0}(x, t)}{\partial x_{j}}\right) & =\frac{\left|Y^{*}\right|}{|Y|} f(x, t), \quad(x, t) \in \Omega \times\left(0, t_{*}\right), \\
T_{0}(x, t)=\bar{T}(x, t), \quad(x, t) \in \Gamma \times\left(0, t_{*}\right), \\
T_{0}(x, 0)=T_{\text {in }}(x), \quad x \in \Omega,
\end{array}\right.
$$

where

$$
\hat{k}_{i j}(x)=\frac{1}{|Y|} \int_{Y^{*}}\left(k_{i p}(x, y) \frac{\partial N_{j}(x, y)}{\partial y_{p}}+k_{i j}(x, y)\right) d y,
$$

$|Y|$ denotes the Lebesgue measure of $Y$. According to the Ref. [2], it can be proved that $\hat{k}_{i j}(x)$ is symmetric, bounded and positive definite for any fixed macroscopic variable $x$.

Remark 3.1. From the conclusions presented in Refs. $[15,16]$, it is easy to prove that the homogenized problems (3.27) have the unique solution $T_{0}(x, t)$.

Meanwhile, combining (3.14) and (3.20), one obtains that

$$
R_{1}(x, y, t)=4 e \sigma T_{0}^{3}(x, t) N_{\alpha_{1}}(x, y) \frac{\partial T_{0}}{\partial x_{\alpha_{1}}}+(1-e) \int_{\Gamma^{c}} R_{1}(x, s, t) F(y, s) d s .
$$

Similarly to (3.20), the solution of $R_{1}(x, y, t)$ can be defined in the following form

$$
R_{1}(x, y, t)=M_{\alpha_{1}}(x, y) T_{0}^{3}(x, t) \frac{\partial T_{0}}{\partial x_{\alpha_{1}}} .
$$

And the auxiliary function $M_{\alpha_{1}}(x, y)$ satisfies the equality

$$
M_{\alpha_{1}}(x, y)=4 e \sigma N_{\alpha_{1}}(x, y)+(1-e) \int_{\Gamma^{c}} M_{\alpha_{1}}(x, s) F(y, s) d s .
$$

$M_{\alpha_{1}}(x, y)$ can be uniquely determined, see Refs. $[13,15]$ for details.

\subsection{Second-order multiscale approximate solutions}

Further, $f(x, t)$ can be substituted by (3.27), to satisfy (3.22), we seek a reasonable expression for $T_{2}(x, y, t)$

$$
T_{2}(x, y, t)=N_{\alpha_{1} \alpha_{2}}(x, y) \frac{\partial^{2} T_{0}}{\partial x_{\alpha_{1}} \partial x_{\alpha_{2}}}+B_{\alpha_{1}}(x, y) \frac{\partial T_{0}}{\partial x_{\alpha_{1}}}+C_{\alpha_{1}}(x, y) T_{0}^{3} \frac{\partial T_{0}}{\partial x_{\alpha_{1}}},
$$

where $T_{0}(x, t)$ is the homogenization solution on $\Omega . N_{\alpha_{1} \alpha_{2}}(x, y), B_{\alpha_{1}}(x, y)$ and $C_{\alpha_{1}}(x, y)$ $\left(\alpha_{1}, \alpha_{2}=1,2,3\right)$ are the local functions defined on $Y^{*}$. One can define them as follows: 
$N_{\alpha_{1} \alpha_{2}}(x, y)$ is the solution of the following problem:

$$
\left\{\begin{array}{l}
\frac{\partial}{\partial y_{i}}\left(k_{i j}(x, y) \frac{\partial N_{\alpha_{1} \alpha_{2}}(x, y)}{\partial y_{j}}\right)=\bar{k}_{\alpha_{1} \alpha_{2}}(x)-\frac{\partial}{\partial y_{i}}\left(k_{i \alpha_{2}}(x, y) N_{\alpha_{1}}(x, y)\right) \\
\quad-k_{\alpha_{1} \alpha_{2}}(x, y)-k_{\alpha_{2} j}(x, y) \frac{\partial N_{\alpha_{1}}(x, y)}{\partial y_{j}}, \quad \text { in } Y^{*}, \\
-v_{i}\left(k_{i j}(x, y) \frac{\partial N_{\alpha_{1} \alpha_{2}}(x, y)}{\partial y_{j}}+k_{i \alpha_{2}}(x, y) N_{\alpha_{1}}(x, y)\right)=0, \quad y \in \Gamma^{c}, \\
N_{\alpha_{1} \alpha_{2}}(x, y) \text { is Y-periodic; }
\end{array}\right.
$$

$B_{\alpha_{1}}(x, y)$ is the solution of the following problem:

$$
\left\{\begin{array}{l}
\frac{\partial}{\partial y_{i}}\left(k_{i j}(x, y) \frac{\partial B_{\alpha_{1}}(x, y)}{\partial y_{j}}\right)=\frac{\partial \bar{k}_{i \alpha_{1}}(x)}{\partial x_{i}}-\frac{\partial k_{i \alpha_{1}}(x, y)}{\partial x_{i}} \\
\quad-\frac{\partial}{\partial x_{i}}\left(k_{i j}(x, y) \frac{\partial N_{\alpha_{1}}(x, y)}{\partial y_{j}}\right)-\frac{\partial}{\partial y_{i}}\left(k_{i j}(x, y) \frac{\partial N_{\alpha_{1}}(x, y)}{\partial x_{j}}\right), \text { in } Y^{*}, \\
-v_{i}\left(k_{i j}(x, y) \frac{\partial B_{\alpha_{1}}(x, y)}{\partial y_{j}}+k_{i j}(x, y) \frac{\partial N_{\alpha_{1}}(x, y)}{\partial x_{j}}\right)=0, \quad y \in \Gamma^{c}, \\
B_{\alpha_{1}}(x, y) \text { is Y-periodic; }
\end{array}\right.
$$

$C_{\alpha_{1}}(x, y)$ is the solution of the following problem:

$$
\left\{\begin{array}{l}
\frac{\partial}{\partial y_{i}}\left(k_{i j}(x, y) \frac{\partial C_{\alpha_{1}}(x, y)}{\partial y_{j}}\right)=0, \quad \text { in } Y^{*}, \\
-v_{i}\left(k_{i j}(x, y) \frac{\partial C_{\alpha_{1}}(x, y)}{\partial y_{j}}\right) \\
\quad=4 e \sigma N_{\alpha_{1}}(x, y)-e \int_{\Gamma^{c}} M_{\alpha_{1}}(x, s) F(y, s) d s, y \in \Gamma^{c}, \\
C_{\alpha_{1}}(x, y) \text { is } Y \text {-periodic, }
\end{array}\right.
$$

where $\bar{k}_{\alpha_{1} \alpha_{2}}=\frac{|Y|}{\left|Y^{*}\right|} \hat{k}_{\alpha_{1} \alpha_{2}}$.

Remark 3.2. Following the idea of Refs. [27-29] and based on the assumptions (B1)-(B4), the cell problems (3.32)-(3.34) have the unique solution for any fixed macroscopic position $x$.

In summary, the multiscale approximate solutions of problems (2.3) are defined as 
follows:

$$
\begin{aligned}
T_{1}^{\varepsilon}(x, t)= & T_{0}+\varepsilon N_{\alpha_{1}}(x, y) \frac{\partial T_{0}}{\partial x_{\alpha_{1}}} \\
T_{2}^{\varepsilon}(x, t)= & T_{0}+\varepsilon N_{\alpha_{1}}(x, y) \frac{\partial T_{0}}{\partial x_{\alpha_{1}}} \\
& +\varepsilon^{2}\left(N_{\alpha_{1} \alpha_{2}}(x, y) \frac{\partial^{2} T_{0}}{\partial x_{\alpha_{1}} \partial x_{\alpha_{2}}}+B_{\alpha_{1}}(x, y) \frac{\partial T_{0}}{\partial x_{\alpha_{1}}}+C_{\alpha_{1}}(x, y) T_{0}^{3} \frac{\partial T_{0}}{\partial x_{\alpha_{1}}}\right),
\end{aligned}
$$

where $T_{1}^{\varepsilon}(x, t)$ and $T_{2}^{\varepsilon}(x, t)$ are called the first-order and the second-order multiscale approximate solutions, respectively. It is worth noting that the expansions (3.35) are different from the traditional forms given by [14,17], the differences are that the correction terms $N_{\alpha_{1}}(x, y), N_{\alpha_{1} \alpha_{2}}(x, y), B_{\alpha_{1}}(x, y)$ and $C_{\alpha_{1}}(x, y)$ are constructed into the asymptotic expansions depending on the macroscopic variable $x$ due to quasi-periodic effect of the materials.

To sum up, one obtains the following theorem.

Theorem 3.1. Temperature fields for the heat conduction-radiation problems (2.3) of porous materials with quasi-periodic structure have the multiscale asymptotic expansions as follows:

$$
\begin{aligned}
T_{\varepsilon}(x, t)= & T_{0}+\varepsilon N_{\alpha_{1}}(x, y) \frac{\partial T_{0}}{\partial x_{\alpha_{1}}}+\varepsilon^{2}\left(N_{\alpha_{1} \alpha_{2}}(x, y) \frac{\partial^{2} T_{0}}{\partial x_{\alpha_{1}} \partial x_{\alpha_{2}}}+B_{\alpha_{1}}(x, y) \frac{\partial T_{0}}{\partial x_{\alpha_{1}}}\right. \\
& \left.+C_{\alpha_{1}}(x, y) T_{0}^{3} \frac{\partial T_{0}}{\partial x_{\alpha_{1}}}\right)+\varepsilon^{3} P_{1}(\varepsilon, x, y, t),
\end{aligned}
$$

where $T_{0}$ is the solution of the homogenized (3.27) with the parameters (3.28). $P_{1}(\varepsilon, x, y, t)$ is the asymptotic expansion function depending on the two-scale variables $x$ and $y . N_{\alpha_{1}}(x, y)$, $N_{\alpha_{1} \alpha_{2}}(x, y), B_{\alpha_{1}}(x, y)$ and $C_{\alpha_{1}}(x, y)$ are the local solutions satisfying (3.21) and (3.32)-(3.34), respectively.

Finally, the approximate temperature gradients can be evaluated by:

$$
\begin{aligned}
\frac{\partial T_{2}^{\varepsilon}(x, t)}{\partial x_{i}}= & \frac{\partial T_{0}}{\partial x_{i}}+\frac{\partial N_{\alpha_{1}}(x, y)}{\partial y_{i}} \frac{\partial T_{0}}{\partial x_{\alpha_{1}}}+\varepsilon \frac{\partial}{\partial x_{i}}\left(N_{\alpha_{1}}(x, y) \frac{\partial T_{0}}{\partial x_{\alpha_{1}}}\right) \\
& +\varepsilon \frac{\partial N_{\alpha_{1} \alpha_{2}}(x, y)}{\partial y_{i}} \frac{\partial^{2} T_{0}}{\partial x_{\alpha_{1}} \partial x_{\alpha_{2}}}+\varepsilon^{2} \frac{\partial}{\partial x_{i}}\left(N_{\alpha_{1} \alpha_{2}}(x, y) \frac{\partial^{2} T_{0}}{\partial x_{\alpha_{1}} \partial x_{\alpha_{2}}}\right) \\
& +\varepsilon \frac{\partial B_{\alpha_{1}}(x, y)}{\partial y_{i}} \frac{\partial T_{0}}{\partial x_{\alpha_{1}}}+\varepsilon^{2} \frac{\partial}{\partial x_{i}}\left(B_{\alpha_{1}}(x, y) \frac{\partial T_{0}}{\partial x_{\alpha_{1}}}\right)+\varepsilon \frac{\partial C_{\alpha_{1}}(x, y)}{\partial y_{i}} T_{0}^{3} \frac{\partial T_{0}}{\partial x_{\alpha_{1}}} \\
& +\varepsilon^{2} \frac{\partial}{\partial x_{i}}\left(C_{\alpha_{1}}(x, y) T_{0}^{3} \frac{\partial T_{0}}{\partial x_{\alpha_{1}}}\right) .
\end{aligned}
$$




\section{Main convergence theorem and its proof}

In this section, the detailed proofs of the explicit convergence order for the second-order multiscale methods in integral sense are given. In addition, in order to get the error estimates of proposed methods, a lemma that will be useful in the sequel is presented at first.

Lemma 4.1. let $g(x, y) \in \hat{L}\left(\Omega \times \mathrm{R}^{n}\right), \int_{Y} g(x, y) d y=0, \forall x \in \Omega$, then

$$
\left|\int_{\Omega} u v g\left(x, \frac{x}{\varepsilon}\right) d x\right| \leqslant C \varepsilon\|u\|_{H^{1}(\Omega)}\|v\|_{H^{1}(\Omega)}
$$

$\forall u, v \in H^{1}(\Omega), C>0$ and is independent $\varepsilon, u, v$.

See the proof in Lemma 1.6 of Ref. [2].

Then, we have the following theorem.

Theorem 4.1. Assume that $\Omega^{\varepsilon} \subset \mathrm{R}^{3}$ is a bounded Lipschitz domain and $\partial \Omega \in C^{4}$. Let $T_{\varepsilon}(x, t)$ be the solution of (2.3), $T_{0}(x, t)$ is the solution of homogenized equation of $(3.27) . T_{2}^{\varepsilon}(x, t)$ is the approximate solution stated in (3.35). Under assumptions (B1)-(B4), if $f(x, t) \in H^{2,1}(\Omega \times$ $\left.\left[0, t_{*}\right)\right), T_{i n}(x) \in H^{4}(\Omega)$, we obtain the following error estimates:

$$
\sup _{0 \leqslant t \leqslant t_{*}} \int_{\Omega^{\varepsilon}}\left(T_{\mathcal{\varepsilon}}(x, t)-T_{2}^{\varepsilon}(x, t)\right)^{2} d x+\int_{0}^{t_{*}}\left\|T_{\mathcal{\varepsilon}}(x, t)-T_{2}^{\varepsilon}(x, t)\right\|_{H^{1}\left(\Omega^{\varepsilon}\right)}^{2} d t \leqslant C \varepsilon,
$$

$C$ is positive constant independent of $\varepsilon$.

Proof. Substituting $T_{\varepsilon}(x, t)-T_{2}^{\varepsilon}(x, t)$ into (2.3), we obtain that

$$
\begin{aligned}
L_{\varepsilon}\left(T_{\varepsilon}(x, t)-T_{2}^{\varepsilon}(x, t)\right)= & \frac{\partial T_{\varepsilon}(x, t)}{\partial t}-\frac{\partial}{\partial x_{i}}\left(k_{i j}(x, y) \frac{\partial T_{\varepsilon}(x, t)}{\partial x_{j}}\right) \\
& -\frac{\partial}{\partial t}\left(T_{0}+\varepsilon N_{\alpha_{1}}(x, y) \frac{\partial T_{0}}{\partial x_{\alpha_{1}}}+\varepsilon^{2}\left(N_{\alpha_{1} \alpha_{2}}(x, y) \frac{\partial^{2} T_{0}}{\partial x_{\alpha_{1}} \partial x_{\alpha_{2}}}+B_{\alpha_{1}}(x, y) \frac{\partial T_{0}}{\partial x_{\alpha_{1}}}\right.\right. \\
& \left.\left.+C_{\alpha_{1}}(x, y) T_{0}^{3} \frac{\partial T_{0}}{\partial x_{\alpha_{1}}}\right)\right)+\frac{\partial}{\partial x_{i}}\left(k _ { i j } ( x , y ) \frac { \partial } { \partial x _ { j } } \left(T_{0}+\varepsilon N_{\alpha_{1}}(x, y) \frac{\partial T_{0}}{\partial x_{\alpha_{1}}}\right.\right. \\
& \left.\left.+\varepsilon^{2}\left(N_{\alpha_{1} \alpha_{2}}(x, y) \frac{\partial^{2} T_{0}}{\partial x_{\alpha_{1}} \partial x_{\alpha_{2}}}+B_{\alpha_{1}}(x, y) \frac{\partial T_{0}}{\partial x_{\alpha_{1}}}+C_{\alpha_{1}}(x, y) T_{0}^{3} \frac{\partial T_{0}}{\partial x_{\alpha_{1}}}\right)\right)\right) \\
= & G_{0}+\varepsilon F_{0}+\varepsilon^{2} \frac{\partial}{\partial x_{i}} F_{i}
\end{aligned}
$$

where

$$
\begin{aligned}
& L_{\varepsilon}=\frac{\partial}{\partial t}-\frac{\partial}{\partial x_{i}}\left(k_{i j}(x, y) \frac{\partial}{\partial x_{j}}\right), \\
& G_{0}=\frac{\partial}{\partial x_{i}}\left(\hat{k}_{i j}(x)-k_{i j}(x, y)-k_{i p}(x, y) \frac{\partial N_{j}(x, y)}{\partial y_{p}}\right) \frac{\partial T_{0}}{\partial x_{\alpha_{1}}},
\end{aligned}
$$




$$
\begin{aligned}
F_{0}= & \frac{\partial}{\partial x_{i}}\left(k_{i j}(x, y) \frac{\partial}{\partial x_{j}}\left(N_{\alpha_{1}}(x, y) \frac{\partial T_{0}}{\partial x_{\alpha_{1}}}\right)\right)+\frac{\partial}{\partial x_{i}}\left(k_{i j}(x, y) \frac{\partial N_{\alpha_{1} \alpha_{2}}(x, y)}{\partial y_{j}} \frac{\partial^{2} T_{0}}{\partial x_{\alpha_{1}} \partial x_{\alpha_{2}}}\right) \\
& +\frac{\partial}{\partial y_{i}}\left(k_{i j}(x, y) \frac{\partial}{\partial x_{j}}\left(N_{\alpha_{1} \alpha_{2}}(x, y) \frac{\partial^{2} T_{0}}{\partial x_{\alpha_{1}} \partial x_{\alpha_{2}}}\right)\right)+\frac{\partial}{\partial x_{i}}\left(k_{i j}(x, y) \frac{\partial B_{\alpha_{1}}(x, y)}{\partial y_{j}} \frac{\partial T_{0}}{\partial x_{\alpha_{1}}}\right) \\
& +\frac{\partial}{\partial y_{i}}\left(k_{i j}(x, y) \frac{\partial}{\partial x_{j}}\left(B_{\alpha_{1}}(x, y) \frac{\partial T_{0}}{\partial x_{\alpha_{1}}}\right)\right)+\frac{\partial}{\partial x_{i}}\left(k_{i j}(x, y) \frac{\partial C_{\alpha_{1}}(x, y)}{\partial y_{j}} T_{0}^{3} \frac{\partial T_{0}}{\partial x_{\alpha_{1}}}\right) \\
& +\frac{\partial}{\partial y_{i}}\left(k_{i j}(x, y) \frac{\partial}{\partial x_{j}}\left(C_{\alpha_{1}}(x, y) T_{0}^{3} \frac{\partial T_{0}}{\partial x_{\alpha_{1}}}\right)\right)-\frac{\partial}{\partial t}\left(N_{\alpha_{1}}(x, y) \frac{\partial T_{0}}{\partial x_{\alpha_{1}}}\right. \\
& \left.+\varepsilon\left(N_{\alpha_{1} \alpha_{2}}(x, y) \frac{\partial^{2} T_{0}}{\partial x_{\alpha_{1}} \partial x_{\alpha_{2}}}+B_{\alpha_{1}}(x, y) \frac{\partial T_{0}}{\partial x_{\alpha_{1}}}+C_{\alpha_{1}}(x, y) T_{0}^{3} \frac{\partial T_{0}}{\partial x_{\alpha_{1}}}\right)\right) \\
F_{i}= & k_{i j}(x, y) \frac{\partial}{\partial x_{j}}\left(C_{\alpha_{1}}(x, y) T_{0}^{3} \frac{\partial T_{0}}{\partial x_{\alpha_{1}}}\right)+k_{i j}(x, y) \frac{\partial}{\partial x_{j}}\left(B_{\alpha_{1}}(x, y) \frac{\partial T_{0}}{\partial x_{\alpha_{1}}}\right) \\
& +k_{i j}(x, y) \frac{\partial}{\partial x_{j}}\left(N_{\alpha_{1} \alpha_{2}}(x, y) \frac{\partial^{2} T_{0}}{\partial x_{\alpha_{1}} \partial x_{\alpha_{2}}}\right) .
\end{aligned}
$$

Let

$$
\left\|G_{0}\right\|_{*}=\sup \left\{\left|\left(G_{0}, v\right)_{L^{2}\left(\Omega^{\varepsilon}\right)}\right|: v \in H_{0}^{1}\left(\Omega^{\varepsilon}\right),\|v\|_{H_{0}^{1}\left(\Omega^{\varepsilon}\right)}=1\right\} .
$$

Then, we have that

$$
\begin{aligned}
\left(G_{0}, v\right)_{L^{2}\left(\Omega^{\varepsilon}\right)} & =\int_{\Omega^{\varepsilon}} G_{0} v d x \\
& =\int_{\Omega^{\varepsilon}}\left(\hat{k}_{i j}(x)-k_{i j}(x, y)-k_{i p}(x, y) \frac{\partial N_{j}(x, y)}{\partial y_{p}}\right) \frac{\partial}{\partial x_{i}}\left(\frac{\partial T_{0}}{\partial x_{\alpha_{1}}} v\right) d x .
\end{aligned}
$$

Also, one can define the following equality given by

$$
g(x, y)=\hat{k}_{i j}(x)-k_{i j}(x, y)-k_{i p}(x, y) \frac{\partial N_{j}(x, y)}{\partial y_{p}} .
$$

Thanks to $\int_{Y} g(x, y) d y=0$ and Lemma 4.1, one obtains that

$$
\left|\int_{\Omega^{\varepsilon}} g\left(x, \frac{x}{\varepsilon}\right) \frac{\partial}{\partial x_{i}}\left(\frac{\partial T_{0}}{\partial x_{\alpha}} v\right) d x\right| \leq C \varepsilon\left\|T_{0}\right\|_{H^{2}(\Omega)}\|v\|_{H^{1}\left(\Omega^{\varepsilon}\right)} .
$$

Obviously, combining (4.6) and (4.8), we obtain

$$
\left\|G_{0}\right\|_{*} \leq C \varepsilon\left\|T_{0}\right\|_{H^{2}(\Omega)} .
$$

For $(x, t) \in \Gamma \times\left(0, t_{*}\right)$, this leads to

$$
\begin{aligned}
T_{\varepsilon}(x, t)-T_{2}^{\varepsilon}(x, t)= & -\varepsilon N_{\alpha_{1}}(x, y) \frac{\partial T_{0}}{\partial x_{\alpha_{1}}}-\varepsilon^{2} N_{\alpha_{1} \alpha_{2}}(x, y) \frac{\partial^{2} T_{0}}{\partial x_{\alpha_{1}} \partial x_{\alpha_{2}}} \\
& -\varepsilon^{2} B_{\alpha_{1}}(x, y) \frac{\partial T_{0}}{\partial x_{\alpha_{1}}}-\varepsilon^{2} C_{\alpha_{1}}(x, y) T_{0}^{3} \frac{\partial T_{0}}{\partial x_{\alpha_{1}}}=\varphi_{\varepsilon}(x, t) .
\end{aligned}
$$


Following the lines of the proof in Theorem 1.2 of (Ref. [2], Chap. II), for any fixed $t \in$ $\left(0, t_{*}\right)$, we obtain

$$
\left\|\varphi_{\varepsilon}(x, t)\right\|_{H^{1 / 2}(\Gamma)} \leq C \varepsilon^{1 / 2}\left\|T_{0}\right\|_{H^{3}(\Omega)} .
$$

where $C$ is a constant independent of $\varepsilon$.

Also for the initial conditions, it follows that

$$
\begin{aligned}
T_{\varepsilon}(x, 0)-T_{2}^{\varepsilon}(x, 0)= & T_{\text {in }}(x)-T_{\text {in }}(x)-\left.\varepsilon N_{\alpha_{1}}(x, y) \frac{\partial T_{0}}{\partial x_{\alpha_{1}}}\right|_{t=0}-\varepsilon^{2}\left(N_{\alpha_{1} \alpha_{2}}(x, y) \frac{\partial^{2} T_{0}}{\partial x_{\alpha_{1}} \partial x_{\alpha_{2}}}\right. \\
& +B_{\alpha_{1}}(x, y) \frac{\partial T_{0}}{\partial x_{\alpha_{1}}}+C_{\alpha_{1}}(x, y) T_{0}^{3} \frac{\partial T_{0}}{\partial x_{\alpha_{1}}} \\
= & \varepsilon \varphi_{0}(x, y), \quad\left\|\varphi_{0}(x, y)\right\|_{L^{2}\left(\Omega^{\varepsilon}\right)} \leq C
\end{aligned}
$$

On the boundary $\Gamma_{\varepsilon, m}^{c}$, let $y=x / \varepsilon, s=z / \varepsilon$, and for the sufficiently smooth homogenized solutions $T_{0}(x, t)$, we have

$$
\begin{aligned}
& -v_{i} k_{i j}^{\varepsilon}(x)\left(\frac{\partial T_{\varepsilon}(x, t)}{\partial x_{j}}-\frac{\partial T_{2}^{\varepsilon}(x, t)}{\partial x_{j}}\right) \\
= & \sigma\left(T_{0}+\varepsilon N_{\alpha_{1}}(x, y) \frac{\partial T_{0}}{\partial x_{\alpha_{1}}}+\varepsilon^{2} N_{\alpha_{1} \alpha_{2}}(x, y) \frac{\partial^{2} T_{0}}{\partial x_{\alpha_{1}} \partial x_{\alpha_{2}}}+B_{\alpha_{1}}(x, y) \frac{\partial T_{0}}{\partial x_{\alpha_{1}}}\right. \\
& \left.+\varepsilon^{2} C_{\alpha_{1}}(x, y) T_{0}^{3} \frac{\partial T_{0}}{\partial x_{\alpha_{1}}}+\varepsilon^{3} P_{1}(\varepsilon, x, y, t)\right) \\
& -\sigma \int_{\Gamma^{c}}\left(T_{0}+\varepsilon N_{\alpha_{1}}(x, s) \frac{\partial T_{0}}{\partial x_{\alpha_{1}}}+\varepsilon^{2} N_{\alpha_{1} \alpha_{2}}(x, s) \frac{\partial^{2} T_{0}}{\partial x_{\alpha_{1}} \partial x_{\alpha_{2}}}+\varepsilon^{2} B_{\alpha_{1}}(x, s) \frac{\partial T_{0}}{\partial x_{\alpha_{1}}}\right. \\
& \left.+\varepsilon^{2} C_{\alpha_{1}}(x, s) T_{0}^{3} \frac{\partial T_{0}}{\partial x_{\alpha_{1}}}+\varepsilon^{3} P_{1}(\varepsilon, x, s, t)\right)^{4} F(y, s) d s \\
& +v_{i} k_{i j}(x, y)\left(\frac{\partial T_{0}}{\partial x_{j}}+\frac{\partial N_{\alpha_{1}}(x, y)}{\partial y_{j}} \frac{\partial T_{0}}{\partial x_{\alpha_{1}}}+\varepsilon N_{\alpha_{1}}(x, y) \frac{\partial^{2} T_{0}}{\partial x_{j} \partial x_{\alpha_{1}}}\right. \\
& +\varepsilon \frac{\partial N_{\alpha_{1} \alpha_{2}}(x, y)}{\partial y_{j}} \frac{\partial^{2} T_{0}}{\partial x_{\alpha_{1}} \partial x_{\alpha_{2}}}+\varepsilon \frac{\partial B_{\alpha_{1}}(x, y)}{\partial y_{j}} \frac{\partial T_{0}}{\partial x_{\alpha_{1}}}+\varepsilon^{2} B_{\alpha_{1}}(x, y) \frac{\partial^{2} T_{0}}{\partial x_{j} \partial x_{\alpha_{1}}} \\
& \left.+\varepsilon^{2} N_{\alpha_{1} \alpha_{2}}(x, y) \frac{\partial^{3} T_{0}}{\partial x_{j} \partial x_{\alpha_{1}} \partial x_{\alpha_{2}}}+\varepsilon \frac{\partial C_{\alpha_{1}}(x, y)}{\partial y_{j}} T_{0}^{3} \frac{\partial T_{0}}{\partial x_{\alpha_{1}}}+\varepsilon^{2} C_{\alpha_{1}}(x, y) \frac{\partial}{\partial x_{j}}\left(T_{0}^{3} \frac{\partial T_{0}}{\partial x_{\alpha_{1}}}\right)\right) .
\end{aligned}
$$

By virtue of the boundary conditions on $\Gamma^{c}$ for $N_{\alpha_{1}}(x, y), N_{\alpha_{1} \alpha_{2}}(x, y), B_{\alpha_{1}}(x, y), C_{\alpha_{1}}(x, y)$ and the regularity of $T_{0}(x, t)$ it follows that

$$
-v_{i} k_{i j}^{\varepsilon}(x)\left(\frac{\partial T_{\varepsilon}(x, t)}{\partial x_{j}}-\frac{\partial T_{2}^{\varepsilon}(x, t)}{\partial x_{j}}\right)=\varepsilon^{2} F
$$


where

$$
\begin{aligned}
F= & v_{i} k_{i j}(x, y)\left(N_{\alpha_{1} \alpha_{2}}(x, y) \frac{\partial^{3} T_{0}}{\partial x_{j} \partial x_{\alpha_{1}} \partial x_{\alpha_{2}}}+B_{\alpha_{1}}(x, y) \frac{\partial^{2} T_{0}}{\partial x_{j} \partial x_{\alpha_{1}}}+C_{\alpha_{1}}(x, y) \frac{\partial}{\partial x_{j}}\left(T_{0}^{3} \frac{\partial T_{0}}{\partial x_{\alpha_{1}}}\right)\right) \\
& +4 T_{0}^{3} \sigma\left(N_{\alpha_{1} \alpha_{2}}(x, y) \frac{\partial^{2} T_{0}}{\partial x_{\alpha_{1}} \partial x_{\alpha_{2}}}+B_{\alpha_{1}}(x, y) \frac{\partial T_{0}}{\partial x_{\alpha_{1}}}+C_{\alpha_{1}}(x, y) T_{0}^{3} \frac{\partial T_{0}}{\partial x_{\alpha_{1}}}\right) \\
& -\int_{\Gamma^{c}} 4 T_{0}^{3} \sigma\left(N_{\alpha_{1} \alpha_{2}}(x, s) \frac{\partial^{2} T_{0}}{\partial x_{\alpha_{1}} \partial x_{\alpha_{2}}}+B_{\alpha_{1}}(x, s) \frac{\partial T_{0}}{\partial x_{\alpha_{1}}}+C_{\alpha_{1}}(x, s) T_{0}^{3} \frac{\partial T_{0}}{\partial x_{\alpha_{1}}}\right) F(y, s) d s \\
& +6 T_{0}^{2} \sigma\left(N_{\alpha_{1}}(x, y) \frac{\partial T_{0}}{\partial x_{\alpha_{1}}}\right)^{2}-\int_{\Gamma^{c}} 6 T_{0}^{2} \sigma\left(N_{\alpha_{1}}(x, s) \frac{\partial T_{0}}{\partial x_{\alpha_{1}}}\right)^{2} F(y, s) d s+\mathcal{O}(\varepsilon) .
\end{aligned}
$$

Thus, combining (4.1), (4.9), (4.10), (4.12) and (4.14), we conclude that $T_{\varepsilon}(x, t)-T_{2}^{\varepsilon}(x, t)$ is a weak solution of the following initial-boundary value problems

$$
\left\{\begin{array}{l}
L_{\varepsilon}\left(T_{\varepsilon}(x, t)-T_{2}^{\varepsilon}(x, t)\right) \\
\quad=G_{0}+\varepsilon F_{0}+\varepsilon^{2} \frac{\partial}{\partial x_{i}} F_{i}, \quad(x, t) \in \Omega^{\varepsilon} \times\left(0, t_{*}\right), \\
T_{\varepsilon}(x, t)-T_{2}^{\varepsilon}(x, t)=\varphi_{\varepsilon}(x, t), \quad(x, t) \in \Gamma \times\left(0, t_{*}\right), \\
T_{\varepsilon}(x, 0)-T_{2}^{\varepsilon}(x, 0)=\varepsilon \varphi_{0}(x, y), \quad x \in \Omega^{\varepsilon}, \\
-v_{i} k_{i j}^{\varepsilon}(x) \frac{\partial\left(T_{\varepsilon}(x, t)-T_{2}^{\varepsilon}(x, t)\right)}{\partial x_{j}}=\varepsilon^{2} F, \quad(x, t) \in \Gamma_{\varepsilon}^{c} \times\left(0, t_{*}\right) .
\end{array}\right.
$$

Noting, after integration and summation over all cells, we obtain a remainder term given by

$$
\sum_{m=1}^{m(\varepsilon)}\left|\Gamma_{\varepsilon, m}^{c}\right| \mathcal{O}\left(\varepsilon^{2}\right)=\mathcal{O}\left(\varepsilon^{-d}\right) \mathcal{O}\left(\varepsilon^{d-1}\right) \mathcal{O}\left(\varepsilon^{2}\right)=\mathcal{O}(\varepsilon),
$$

where $d$ is 2 or 3 in actual applications. Then, from the regularity of $N_{\alpha_{1}}(x, y), N_{\alpha_{1} \alpha_{2}}(x, y)$, $B_{\alpha_{1}}(x, y), C_{\alpha_{1}}(x, y), T_{0}(x, t)$, and using Gronwall's inequality, we complete the proof of Theorem 4.1.

\section{Multiscale finite element algorithms}

In this section, we give the detailed algorithm procedure to derive the second-order multiscale finite element formulations of the heat conduction-radiation problems (2.3), and investigate the multiscale behavior of the coupled problems.

\subsection{Multiscale finite element (FE) formulation}

1) FE formulations of auxiliary cell functions and homogenized parameters. 
The FE solutions of auxiliary cell functions $N_{\alpha_{1}}^{h}(x, y)$ can be obtained by solving the following FE virtual work equation on unit cell $Y^{*}$

$$
\int_{Y^{*}} k_{i j}(x, y) \frac{\partial N_{\alpha_{1}}^{h}(x, y)}{\partial y_{j}} \frac{\partial v}{\partial y_{i}} d y=\int_{Y^{*}}-k_{i \alpha_{1}}(x, y) \frac{\partial v}{\partial y_{i}} d y, \quad \forall v \in S^{h}\left(Y^{*}\right)
$$

where $S^{h}\left(Y^{*}\right) \subseteq H_{0}^{1}\left(Y^{*}\right)$ denotes the FE space of 1-square $Y^{*}, Y^{*}$ is partitioned into FE set $S^{h}$ of finite elements, $h$ is the mesh size, as shown in Fig. 2(a). Then, the FE approximation $\hat{k}_{i j}(x)$ of homogenized coefficients can be obtained as follows:

$$
\hat{k}_{i j}^{h}(x)=\frac{1}{|Y|} \int_{Y^{*}}\left(k_{i p}(x, y) \frac{\partial N_{j}^{h}(x, y)}{\partial y_{p}}+k_{i j}(x, y)\right) d y .
$$

Similarly to (5.1) and (5.2), $N_{\alpha_{1} \alpha_{2}}^{h}(x, y), B_{\alpha_{1}}^{h}(x, y), M_{\alpha_{1}}^{h}(x, y)$ and $C_{\alpha_{1}}^{h}(x, y)$ can be given by solving the following equations, successively,

$$
\begin{aligned}
& \int_{Y^{*}} k_{i j}(x, y) \frac{\partial N_{\alpha_{1} \alpha_{2}}^{h}(x, y)}{\partial y_{j}} \frac{\partial v}{\partial y_{i}} d y \\
= & \int_{Y^{*}}\left(k_{\alpha_{1} \alpha_{2}}(x, y)-\bar{k}_{\alpha_{1} \alpha_{2}}^{h}(x)+k_{\alpha_{2} j}(x, y) \frac{\partial N_{\alpha_{1}}^{h}(x, y)}{\partial y_{j}}\right) v d y \\
& -\int_{Y^{*}} k_{i \alpha_{2}}(x, y) N_{\alpha_{1}}^{h}(x, y) \frac{\partial v}{\partial y_{i}} d y, \quad \forall v \in S^{h}\left(Y^{*}\right), \\
& \int_{Y^{*}} k_{i j}(x, y) \frac{\partial B_{\alpha_{1}}^{h}(x, y)}{\partial y_{j}} \frac{\partial v}{\partial y_{i}} d y \\
= & \int_{Y^{*}}\left(\frac{\partial k_{i \alpha_{1}}(x, y)}{\partial x_{i}}-\frac{\partial \bar{k}_{i \alpha_{1}}(x)}{\partial x_{i}}+\frac{\partial}{\partial x_{i}}\left(k_{i j}(x, y) \frac{\partial N_{\alpha_{1}}(x, y)}{\partial y_{j}}\right)\right) v d y \\
& -\int_{Y^{*}} k_{i j}(x, y) \frac{\partial N_{\alpha_{1}}(x, y)}{\partial x_{j}} \frac{\partial v}{\partial y_{i}} d y, \quad \forall v \in S^{h}\left(Y^{*}\right), \\
& M_{\alpha_{1}}^{h}(x, y)=4 e \sigma N_{\alpha_{1}}^{h}(x, y)+(1-e) \int_{\Gamma^{c}} M_{\alpha_{1}}^{h}(x, s) F(y, s) d s, \\
& \int_{Y^{*}} k_{i j}(x, y) \frac{\partial C_{\alpha_{1}}^{h}(x, y)}{\partial y_{j}} \frac{\partial v}{\partial y_{i}} d y \\
= & -\int_{\Gamma^{c}}\left(4 e \sigma N_{\alpha_{1}}^{h}(x, y)-e \int_{\Gamma^{c}} M_{\alpha_{1}}^{h}(x, s) F(y, s) d s\right) v d y, \quad \forall v \in S^{h}\left(Y^{*}\right) .
\end{aligned}
$$

2) FE formulations of homogenized problems.

The FE solutions of the homogenized problems (3.27) are evaluated by following FE 


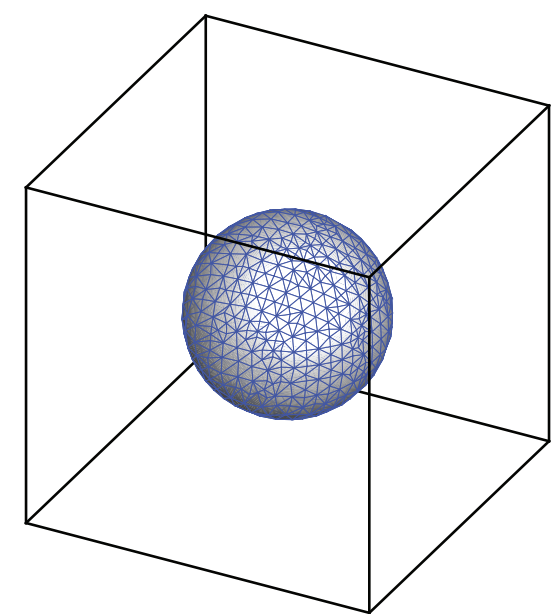

(a)

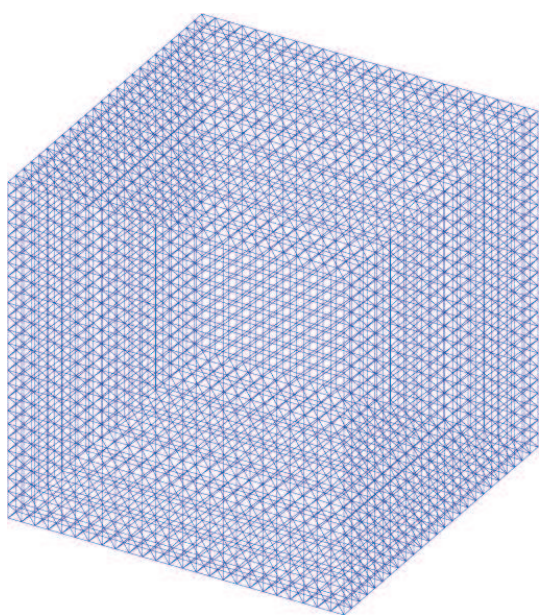

(b)

Figure 2: (a) The meshes of unit cell; (b) the meshes of the homogenized domain $\Omega$.

virtual work equations together with the homogenized coefficients (3.28) on $\Omega$

$$
\begin{aligned}
& \int_{\Omega} \frac{\left|Y^{*}\right|}{|Y|} \frac{\partial T_{0}^{h_{0}}(x, t)}{\partial t} v d x+\int_{\Omega} \hat{k}_{i j}^{h}(x) \frac{\partial T_{0}^{h_{0}}(x, t)}{\partial x_{i}} \frac{\partial v}{\partial x_{j}} d x \\
= & \int_{\Omega} \frac{\left|Y^{*}\right|}{|Y|} f(x, t) v d x, \quad \forall v \in S^{h_{0}}(\Omega),
\end{aligned}
$$

where $S^{h_{0}}(\Omega)$ denotes the FE space with mesh size $h_{0}$ on the homogenized domain $\Omega$, as shown in Fig. 2(b). The backward Euler full discrete format proposed in Refs. $[17,32]$ is used for the discretization.

3) Multiscale finite element solutions.

From (3.35) and (5.1)-(5.7), the multiscale finite element solutions based on whole structure $\Omega^{\varepsilon}$ can be given by

$$
\begin{aligned}
T_{1, h, h_{0}}^{\varepsilon}(x, t)= & T_{0}^{h_{0}}+\varepsilon N_{\alpha_{1}}^{h}(x, y) \frac{\partial T_{0}^{h_{0}}}{\partial x_{\alpha_{1}}} \\
T_{2, h, h_{0}}^{\varepsilon}(x, t)= & T_{0}^{h_{0}}+\varepsilon N_{\alpha_{1}}^{h}(x, y) \frac{\partial T_{0}^{h_{0}}}{\partial x_{\alpha_{1}}} \\
& +\varepsilon^{2}\left(N_{\alpha_{1} \alpha_{2}}^{h}(x, y) \frac{\partial^{2} T_{0}^{h_{0}}}{\partial x_{\alpha_{1}} \partial x_{\alpha_{2}}}+C_{\alpha_{1}}^{h}(x, y)\left(T_{0}^{h_{0}}\right)^{3} \frac{\partial T_{0}^{h_{0}}}{\partial x_{\alpha_{1}}}\right) .
\end{aligned}
$$

\subsection{FE algorithm procedure for second-order multiscale method}

The algorithm procedure of the second-order multiscale method for predicting the thermal properties of the original problems (2.3) is presented as follows: 
1) Verify and generate the distribution of cavities in unit cell, and the geometric model of the structure. Further, partition $Y^{*}$ into finite element meshes set.

2) Compute the auxiliary cell functions $N_{\alpha_{1}}(x, y)\left(\alpha_{1}=1,2,3\right)$ on the reference cell by solving the cell functions problems (5.1) with finite element method from Section 5.1. Then, the homogenized coefficient $\hat{k}_{i j}(x)(i, j=1,2,3)$ can be evaluated by the formula (5.2).

3) Calculate the homogenized solutions $T_{0}(x, t)$ by solving problems (5.7) on global structure $\Omega$ in a coarse mesh and in a large time step, and the standard Backward Euler full discrete format is employed to solve the modified homogenized problems.

4) Solve $N_{\alpha_{1} \alpha_{2}}(x, y), B_{\alpha_{1}}(x, y), M_{\alpha_{1}}(x, y)$ and $C_{\alpha_{1}}(x, y)\left(\alpha_{1}, \alpha_{2}=1,2,3\right)$ by computing the auxiliary cell problems (5.3)-(5.6) with finite element method (FEM) based on reference cell $Y^{*}$, respectively.

5) Compute the spatial variable derivatives $\frac{\partial T_{0}}{\partial x_{\alpha_{1}}}$ and $\frac{\partial^{2} T_{0}}{\partial x_{\alpha_{1}} \partial x_{\alpha_{2}}}\left(\alpha_{1}, \alpha_{2}=1,2,3\right)$ by the average technique on relative elements (see Refs. [29,33]).

6) Use the auxiliary cell functions from steps 2 and 4 to evaluate the multiscale temperature and the temperature gradient fields according to formulas (3.35) and (3.37), respectively.

\section{Numerical examples and discussions}

\subsection{Validation of the second-order multiscale method}

In this subsection, we present some examples to validate the second-order multiscale method proposed previously. Take into account the mixed boundary value problems (2.3), where $\Omega^{\varepsilon}$ consist of whole cells that are illustrated in Fig. 3(a), and the unit cell $Y^{*}$ is illustrated in Fig. 3(b). The boundary temperatures in the $x_{3}$-direction are set as $\bar{T}_{1}=0 \mathrm{~K}$, $\bar{T}_{2}=0 \mathrm{~K}$ and the time step is $\Delta t=0.02 . \sigma=5.669996 \times 10^{-8} \mathrm{~W} / \mathrm{m}^{2} \mathrm{~K}^{4}$, and the radius of the cavity in $Y^{*}$ is 0.25 , respectively. The initial temperature $T_{\text {in }}(x)$ is set to $0.0 \mathrm{~K}$.

Since it is too hard to find the analytical solutions of the original problems (2.3), we have to take $T_{\varepsilon}(x, t)$ to be its FE solution $T_{F E}$ in the extremely fine meshes. The linear tetrahedral elements are applied for the problems of (2.3) using fine meshes and that of the corresponding homogenized equations by a coarse mesh. Table 1 shows the numbers of tetrahedrons and nodes.

Table 1: Comparison of computational cost.

\begin{tabular}{||lccc||}
\hline & Original equation & Unit cell & Homogenized equation \\
\hline Elements & 727125 & 5817 & 93750 \\
Nodes & 168875 & 1351 & 17576 \\
\hline
\end{tabular}




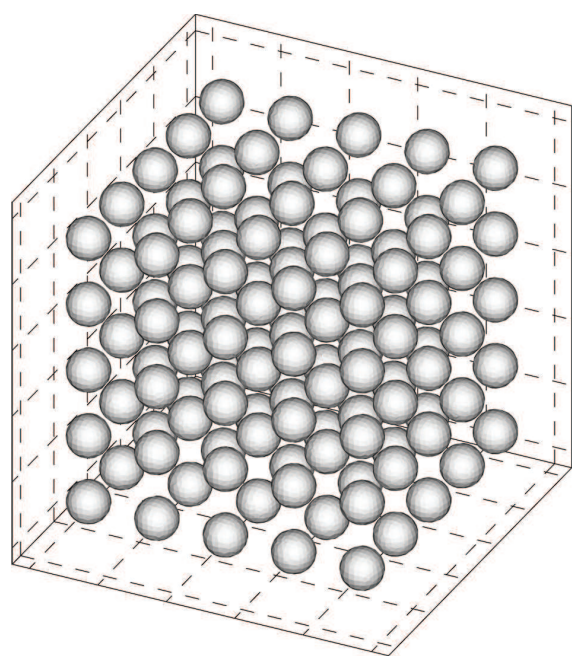

(a)

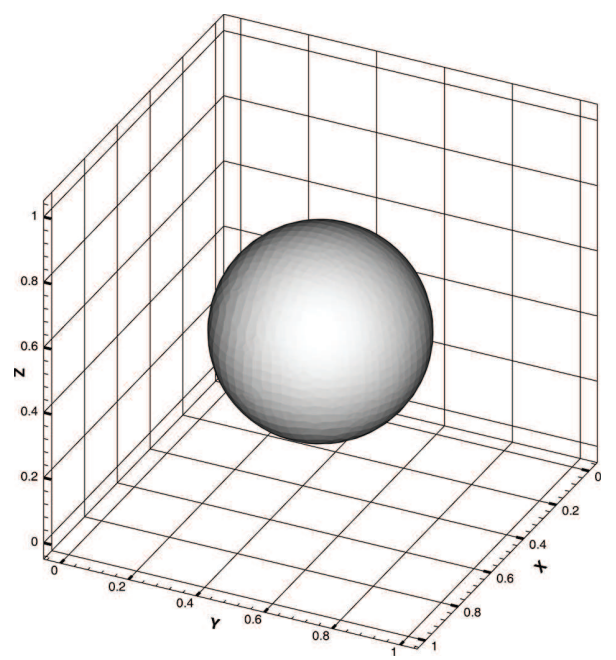

(b)

Figure 3: (a) Domain $\Omega^{\varepsilon}=[0,0.25]^{3}$; (b) Unit cell $Y^{*}=[0,1]^{3}$.

The following cases are investigated:

Case1: $\mathrm{e}=1.0, \omega(x)=1+\frac{1}{5}\left(x_{1}+x_{2}+x_{3}\right), k_{i j}=10 \delta_{i j} \mathrm{~W} / \mathrm{m} \mathrm{K}, f(x, t)=10^{5} \mathrm{~J} / \mathrm{m}^{3} \mathrm{~s} ;$

Case2: $\mathrm{e}=0.2, \omega(x)=1+\frac{1}{5}\left(x_{1}+x_{2}+x_{3}\right), k_{i j}=10 \delta_{i j} \mathrm{~W} / \mathrm{m} \mathrm{K}, f(x, t)=10^{5} \mathrm{~J} / \mathrm{m}^{3} \mathrm{~s}$;

Case3: $\mathrm{e}=1.0, \omega(x)=1+x_{1}+x_{2}+x_{3}, k_{i j}=10 \delta_{i j} \mathrm{~W} / \mathrm{m} \mathrm{K}, f(x, t)=10^{5} \mathrm{~J} / \mathrm{m}^{3} \mathrm{~s}$;

Case4: $\mathrm{e}=1.0, \omega(x)=1+\frac{1}{5}\left(x_{1}^{2}+x_{2}^{2}+x_{3}^{2}\right), k_{i j}=10 \delta_{i j} \mathrm{~W} / \mathrm{m} \mathrm{K}, f(x, t)=10^{5} \mathrm{~J} / \mathrm{m}^{3} \mathrm{~s}$, where $\delta_{i j}(i, j=1,2,3)$ is the Kronecker delta, and if $i=j, \delta_{i j}=1$, or $\delta_{i j}=0 . k_{i j}\left(x, \frac{x}{\varepsilon}\right)=\omega(x) k_{i j}$ $(i, j=1,2,3)$. It should be emphasized that $T_{0}^{h_{0}}(x, t)$ is the FE solution of the homogenized equations (5.7), $T_{1, h, h_{0}}^{\varepsilon}(x, t)$ and $T_{2, h, h_{0}}^{\varepsilon}(x, t)$ denote the FE solution of first-order and the second-order multiscale methods based on (5.8). In addition, we give the following notations

$$
\|v\|_{L^{2}}=\left(\int_{\Omega^{\varepsilon}}|v|^{2} d x\right)^{1 / 2}, \quad|v|_{H^{1}}=\left(\int_{\Omega^{\varepsilon}}\left(|\nabla v|^{2}\right) d x\right)^{1 / 2} .
$$

The relative numerical errors of the homogenized methods, first-order multiscale and second-order multiscale methods in $L^{2}$-norm and $H^{1}$-norm for the examples are listed in Tables 2 and 3, respectively. Also, we define that error $0=T_{F E}-T_{0}^{h_{0}}(x, t)$, error ${ }_{1}=T_{F E}-$ $T_{1, h, h_{0}}^{\varepsilon}(x, t)$ and error $2=T_{F E}-T_{2, h, h_{0}}^{\varepsilon}(x, t)$ to simplify the problems.

Figs. 4(a)-(d) display the computational results for $T_{0}^{h_{0}}(x, t), T_{1, h, h_{0}}^{\varepsilon}(x, t), T_{2, h, h_{0}}^{\varepsilon}(x, t)$ and $T_{F E}$ at the intersection $x_{3}=0.15$ in Case 1 and at time $t=0.2$.

Figs. 5(a)-(d) display the computational results for $T_{0}^{h_{0}}(x, t), T_{1, h, h_{0}}^{\varepsilon}(x, t), T_{2, h, h_{0}}^{\varepsilon}(x, t)$ and $T_{F E}$ at the intersection $x_{3}=0.15$ in Case 4 and at time $t=0.2$. 
Table 2: Comparison with computing results of norm $L^{2}$ and at time $t=0.2$.

\begin{tabular}{||lccc||}
\hline & $\|$ error $_{0}\left\|_{L^{2}} /\right\| T_{F E} \|_{L^{2}}$ & $\|$ error $_{1}\left\|_{L^{2}} /\right\| T_{F E} \|_{L^{2}}$ & $\|$ error $_{2}\left\|_{L^{2}} /\right\| T_{F E} \|_{L^{2}}$ \\
\hline Case1 & 0.01135923 & 0.003123321 & 0.002132161 \\
Case2 & 0.01164171 & 0.004087159 & 0.003306059 \\
Case3 & 0.01135869 & 0.003120886 & 0.002132407 \\
Case4 & 0.01135845 & 0.003122768 & 0.002131599 \\
\hline
\end{tabular}

Table 3: Comparison with computing results of semi-norm $H^{1}$ and at time $t=0.2$.

\begin{tabular}{||lccc||}
\hline & error $\left._{0}\right|_{H^{1}} /\left|T_{F E}\right|_{H^{1}}$ & error $\left._{1}\right|_{H^{1}} /\left|T_{F E}\right|_{H^{1}}$ & error $\left._{2}\right|_{H^{1}} /\left|T_{F E}\right|_{H^{1}}$ \\
\hline Case1 & 0.17172187 & 0.06500094 & 0.063661502 \\
Case2 & 0.17170429 & 0.06530554 & 0.063959088 \\
Case3 & 0.17159739 & 0.06504279 & 0.063744252 \\
Case4 & 0.17171492 & 0.064946702 & 0.063611723 \\
\hline
\end{tabular}

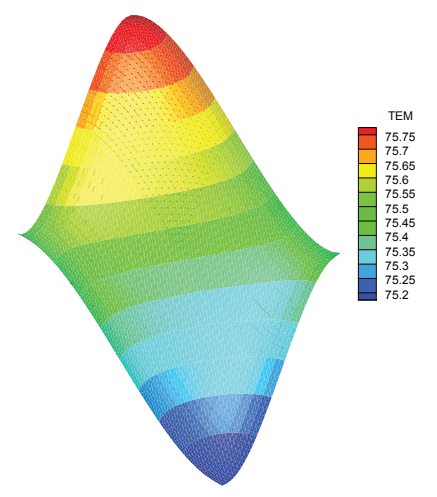

(a)

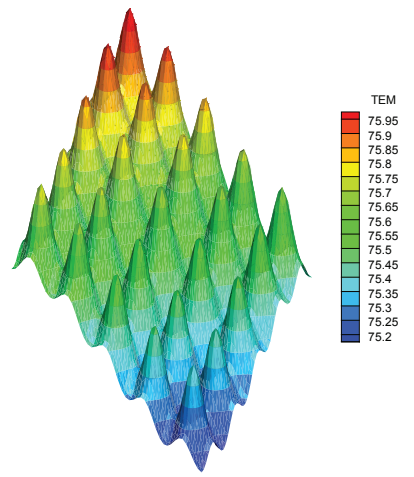

(c)

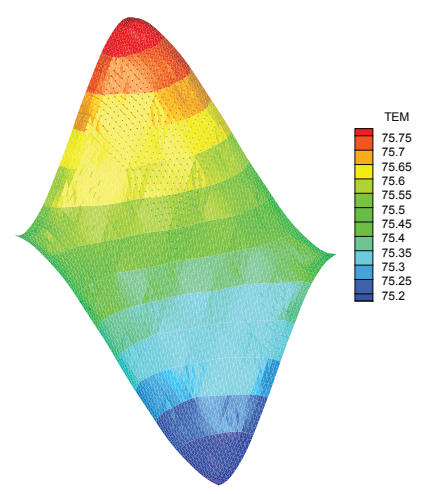

(b)

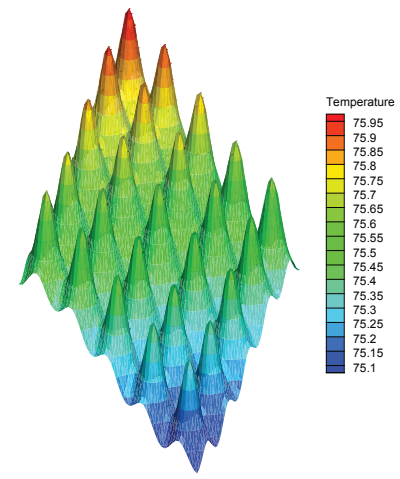

(d)

Figure 4: Temperature fields in the cross section $x_{3}=0.15$ and at time $t=0.2$, Case 1 ; (a) $T_{0}^{h_{0}}(x, t)$; (b) $T_{1, h, h_{0}}^{\varepsilon}(x, t) ;(\mathrm{c}) T_{2, h, h_{0}}^{\varepsilon}(x, t) ;(\mathrm{d}) T_{F E}$. 


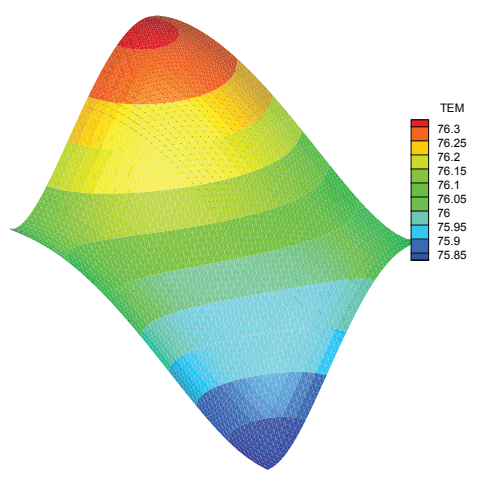

(a)

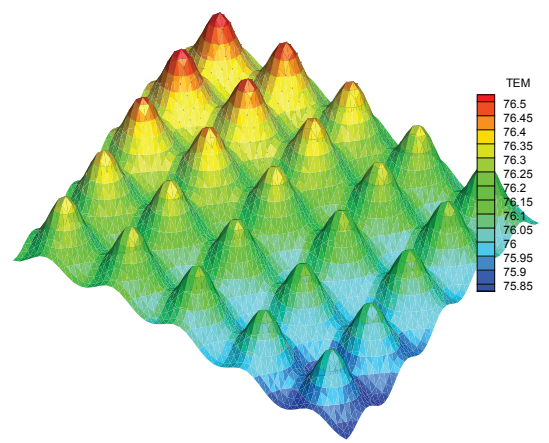

(c)

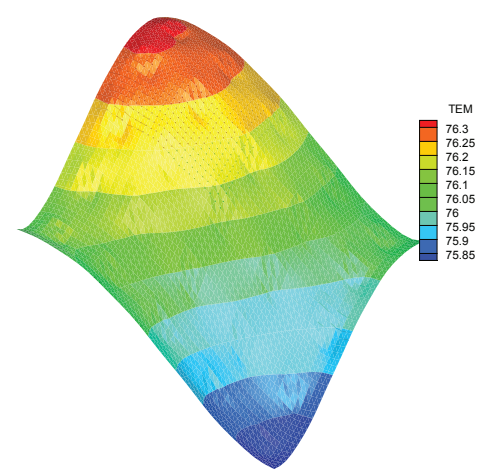

(b)

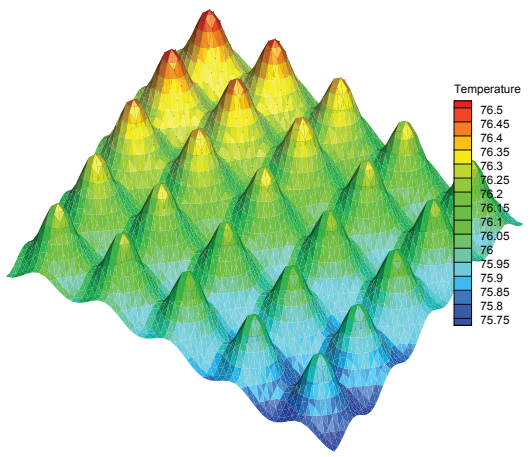

(d)

Figure 5: Temperature fields in the cross section $x_{3}=0.15$ and at time $t=0.2$, Case 4 ; (a) $T_{0}^{h_{0}}(x, t)$; (b) $T_{1, h, h_{0}}^{\varepsilon}(x, t) ;(\mathrm{c}) T_{2, h, h_{0}}^{\varepsilon}(x, t) ;(\mathrm{d}) T_{F E}$.

Figs. 6(a) and (b) illustrate the multiscale finite element results for temperature gradients $\frac{\partial T_{\varepsilon}(x, t)}{\partial x_{1}}, \frac{\partial T_{\varepsilon}(x, t)}{\partial x_{2}}$ along the line of $x_{1}=x_{2}, x_{3}=0.15$ in Case 1 and at time $t=0.2$.

Figs. 7(a) and (b) illustrate the multiscale finite element results for temperature gradients $\frac{\partial T_{\varepsilon}(x, t)}{\partial x_{1}}, \frac{\partial T_{\varepsilon}(x, t)}{\partial x_{2}}$ along the line of $x_{1}=x_{2}, x_{3}=0.15$ in Case 3 and at time $t=0.2$.

The relative errors of multiscale approximate solutions are shown clearly in Figs. 8 and 9 with time $t$ for the Cases 1 and 4, where error0L2, error1L2, error2L2, error0H1, error1H1, and error2H1 denote $\|$ error $_{0}\left\|_{L^{2}} /\right\| T_{F E}\left\|_{L^{2}},\right\|$ error $_{1}\left\|_{L^{2}} /\right\| T_{F E}\left\|_{L^{2}},\right\|$ error $_{2}\left\|_{L^{2}} /\right\| T_{F E} \|_{L^{2}}$, error $\left._{0}\right|_{H^{1}} /\left|T_{F E}\right|_{H^{1}}$, error $\left._{1}\right|_{H^{1}} /\left|T_{F E}\right|_{H^{1}}$, error $\left._{2}\right|_{H^{1}} /\left|T_{F E}\right|_{H^{1}}$, respectively.

By analyzing the numerical results shown in Figs. 4-7, Tables 2 and 3, we found that the homogenized solutions and the first-order solutions are not sufficient to capture the local fluctuation behavior for such coupled problems. And only second-order multiscale solutions can accurately catch the microscale oscillating information. Figs. 8 and 9 display the evolution of relative errors between different multiscale approximate solutions and FE solutions computed on refined meshes. It is worth noting that the relative error does not increase significantly with time. This shows that the multiscale method is a good 


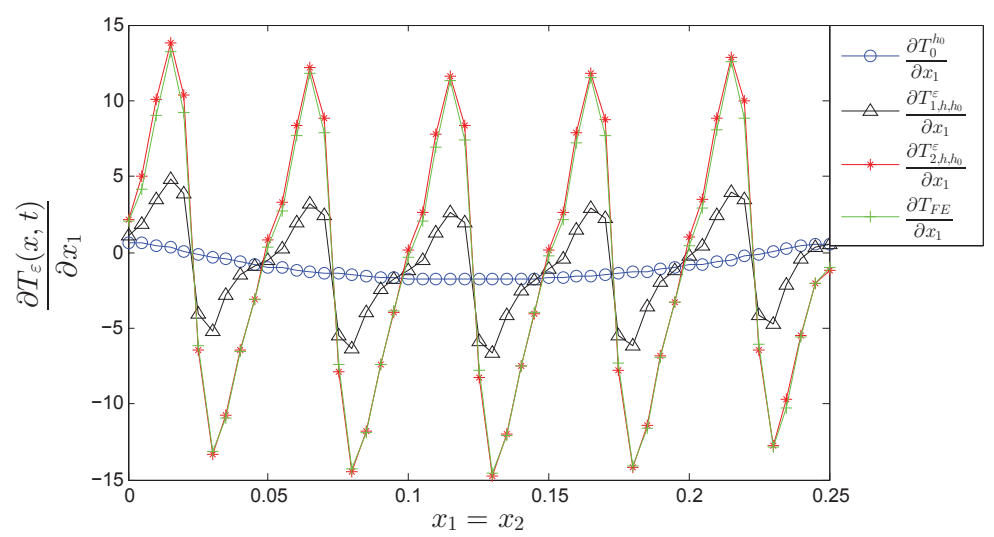

(a)

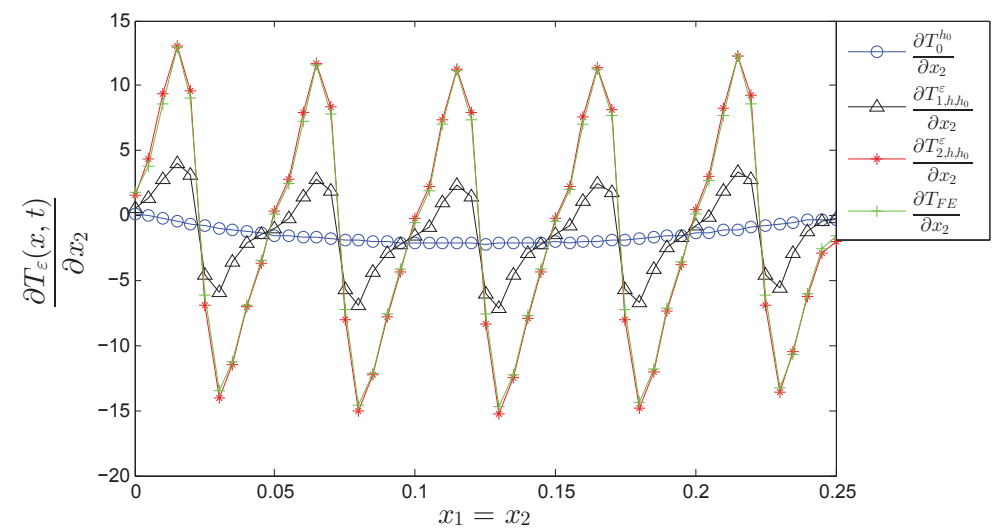

(b)

Figure 6: Case 1 ; (a) $\frac{\partial T_{\varepsilon}(x, t)}{\partial x_{1}}$; (b) $\frac{\partial T_{\varepsilon}(x, t)}{\partial x_{2}}$ along the line of $x_{1}=x_{2}, x_{3}=0.15$ and at time $t=0.2$.

way, in some cases, to deal with a long-time problems. Obviously, the second-order multiscale method proposed in this paper is effective for computing the coupling problems of porous materials with quasi-periodic structures.

In order to verify the influence of radiation effect on the temperature fields, we carry out a numerical example with different boundary temperatures.

The macrostructure $\Omega^{\varepsilon}$ of Fig. 3(a) is chosen in this example, and the emissivity is $e=1$. The internal heat source $f(x, t)$ is set to $10000 \mathrm{~J} / \mathrm{m}^{3} \mathrm{~s}, k_{i j}=0.5 \delta_{i j} \mathrm{~W} / \mathrm{m}$, and $\omega(x)=$ $1+\frac{1}{5}\left(x_{1}+x_{2}+x_{3}\right)$. Figs. 10(a)-(c) show the computing results of the temperature fields $T_{\mathcal{\varepsilon}}(x, t)$ for different boundary temperatures i.e., $\bar{T}_{1}=100 \mathrm{~K}, \bar{T}_{2}=1000 \mathrm{~K} ; \bar{T}_{1}=500 \mathrm{~K}, \bar{T}_{2}=$ $1200 \mathrm{~K} ; \bar{T}_{1}=800 \mathrm{~K}, \bar{T}_{2}=1500 \mathrm{~K}$, and along the line of $x_{1}=0.15, x_{3}=0.15$ and at time $t=0.2$.

As displayed in the Fig. 10, the second-order multiscale approximation is adequately effective to capture the microscopic oscillating behavior of the coupled solutions. Also, the second order expansions developed in this paper can acquire more detailed information about the radiation effect of the cavities in the porous materials. 


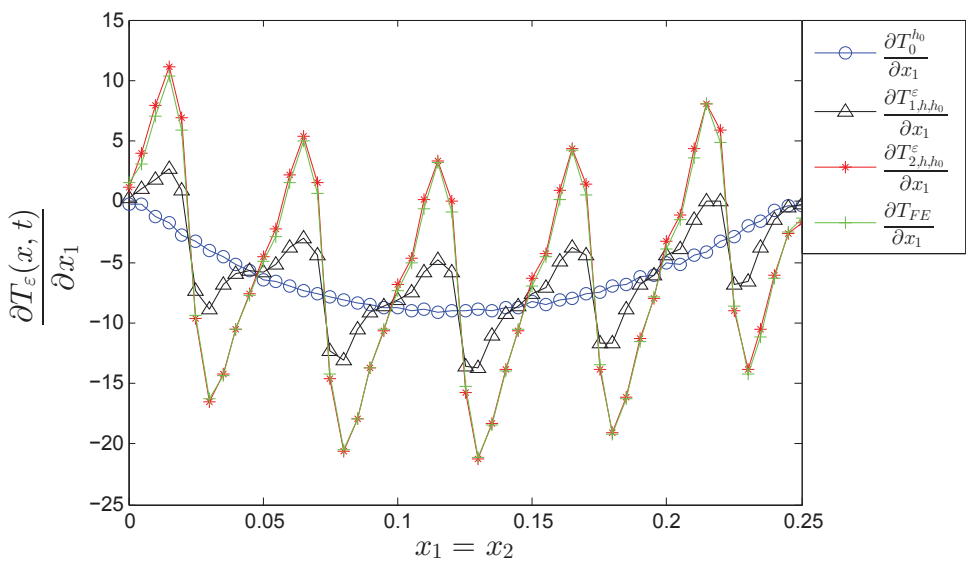

(a)

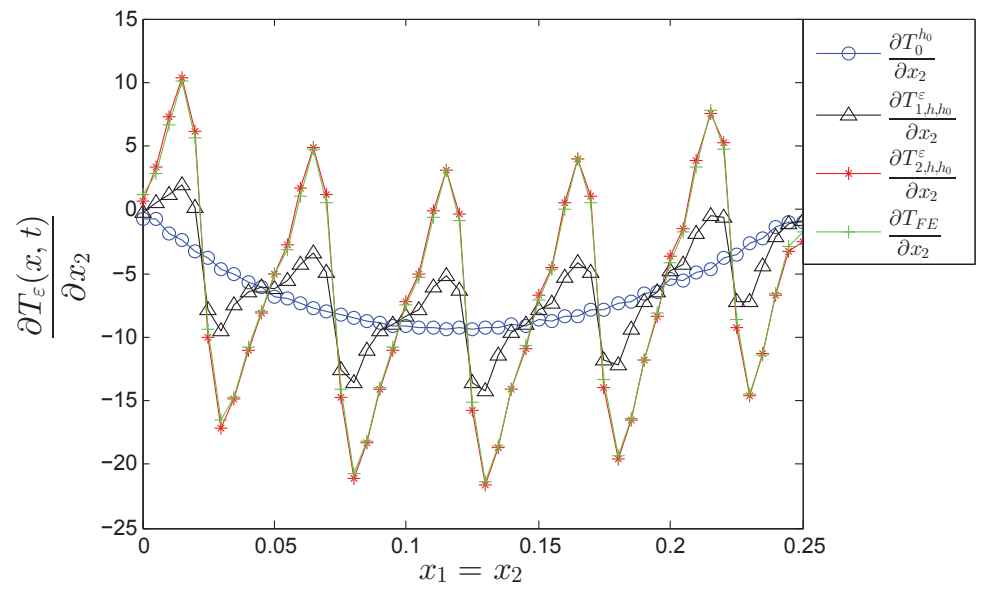

(b)

Figure 7: Case 3; (a) $\frac{\partial T_{\varepsilon}(x, t)}{\partial x_{1}}$; (b) $\frac{\partial T_{\varepsilon}(x, t)}{\partial x_{2}}$ along the line of $x_{1}=x_{2}, x_{3}=0.15$ and at time $t=0.2$.

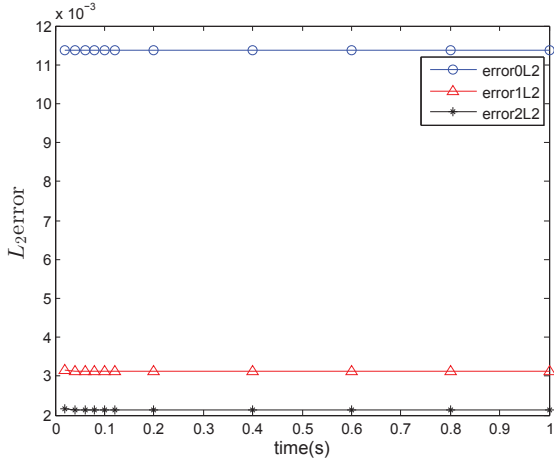

(a)

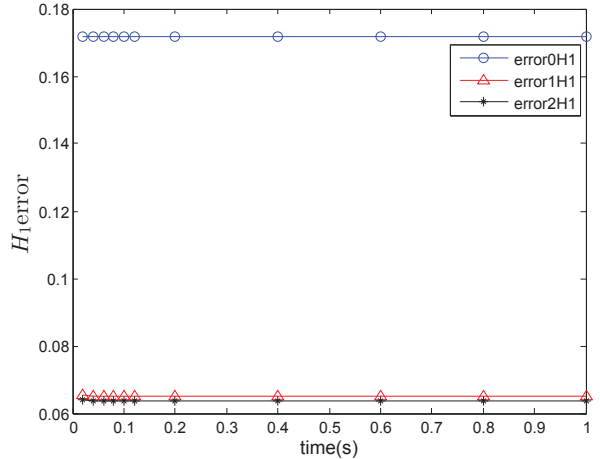

(b)

Figure 8: Case 1; (a) the evolution of $L^{2}$ relative errors with $t$; (b) the evolution of $H^{1}$ relative errors with $t$. 


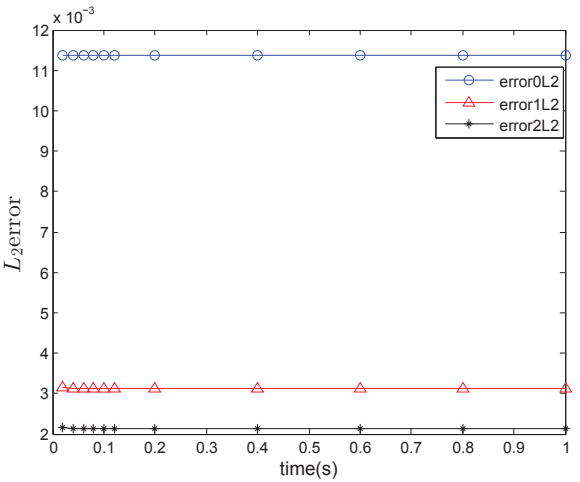

(a)

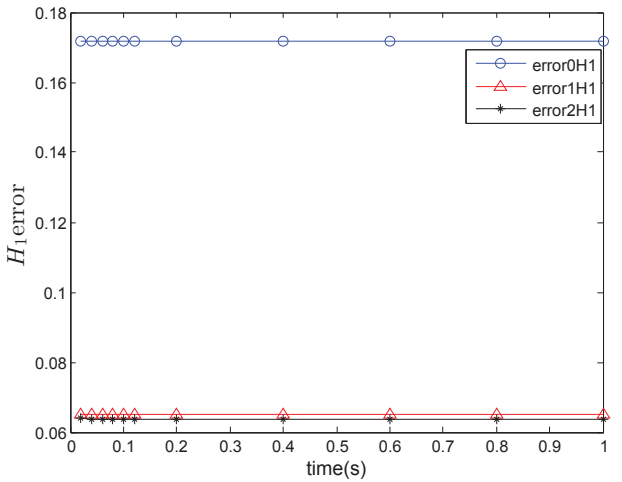

(b)

Figure 9: Case 4; (a) the evolution of $L^{2}$ relative errors with $t$; (b) the evolution of $H^{1}$ relative errors with $t$.

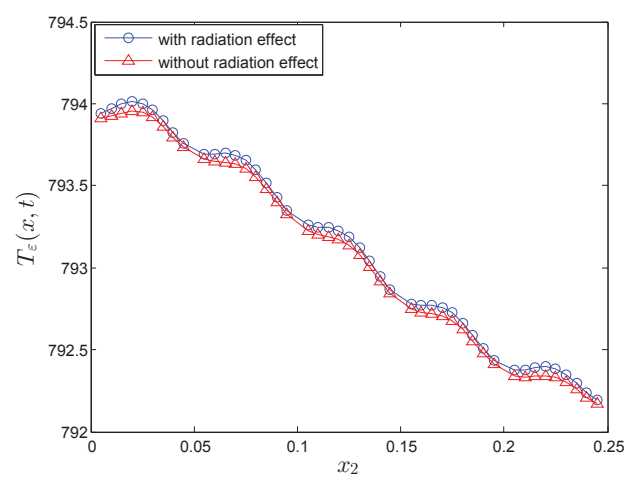

(a)

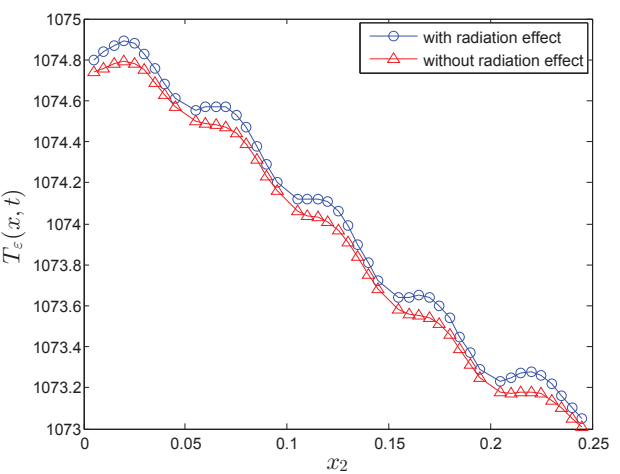

(b)

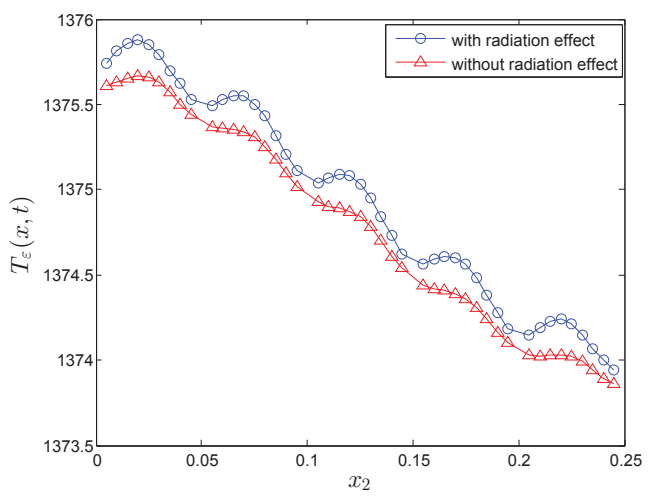

(c)

Figure 10: Temperature fields $T_{\varepsilon}(x, t)$ for different boundary temperatures along the line of $x_{1}=0.15, x_{3}=0.15$ and at time $t=0.2$ : (a) $\bar{T}_{1}=100 \mathrm{~K}, \bar{T}_{2}=1000 \mathrm{~K}$; (b) $\bar{T}_{1}=500 \mathrm{~K}, \bar{T}_{2}=1200 \mathrm{~K}$; (c) $\bar{T}_{1}=800 \mathrm{~K}, \bar{T}_{2}=1500 \mathrm{~K}$. 


\subsection{Porous materials with a large number of pores}

In practical engineering problems, porous materials are composed of a large number of pores. Thus, in this subsection, the porous materials described in Fig. 11 which consist of 200000 pores with a diameter of $d=1.25 \mathrm{~mm}$ are used to demonstrate the effectiveness of the second-order multiscale method. All the pores are also generated by rigid wall constraints in a rectangular region. The thermal conductivity is $10 \mathrm{~W} / \mathrm{mK}, \omega(x)=1+$ $\frac{1}{5}\left(x_{1}+x_{2}+x_{3}\right)$ and boundary conditions are the same as that listed in Section 6.1. The internal heat source is $f(x, t)=10^{5} \mathrm{~J} / \mathrm{m}^{3} \mathrm{~s}$.

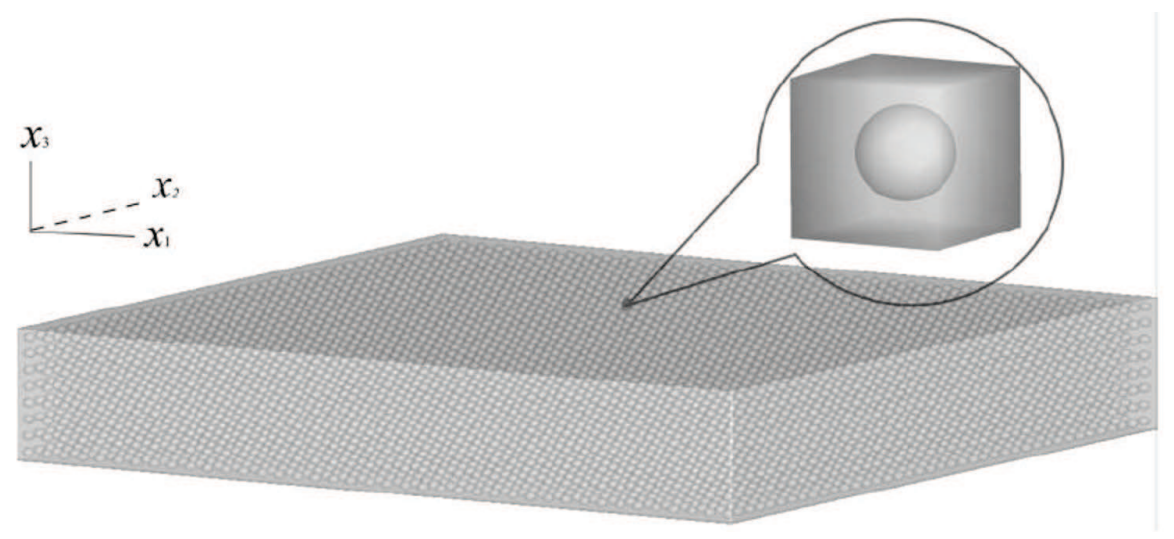

Figure 11: Porous materials with a large number of pores. $\Omega^{\varepsilon}=[0,0.25 \mathrm{~m}]^{2} \times[0,0.2 \mathrm{~m}]$.

Figs. 12(a)-(c) illustrate the partial numerical results for $\frac{\partial T_{\varepsilon}(x, t)}{\partial x}$ along the line of $x_{1}=x_{2}$, $x_{3}=0.15$ and at time $t=0.2$.

Both the second-order multiscale methods and the direct numerical simulations are carried out on the same computer (which has memory of 512GB and 16 processors with $\mathrm{CPU}=2.67 \mathrm{GHz}$ ). On the aspect of the multiscale methods, it is very cheap to solve the simulation (it takes about 1 second to finish solving the cell problems, and homogenized problems about 7 seconds for one iteration). However, we cannot easily obtain $T_{\mathrm{FE}}$ by classical numerical method since it requires very fine meshes and a great amount of computation time, and the convergence of FE method based on fine meshes for the nonlinear coupled problems is also not easy.

As a result, the second-order multiscale approximate solutions developed in this work can effectively capture the local fluctuation caused by the complicated microstructure. Besides, the second-order multiscale method is appropriate for a very small periodic parameter $\varepsilon$, i.e., a large number of pores contained in the porous materials, which can greatly save the computing memory and CPU time without loss of accuracy, and it is very important in actual engineering problems. 


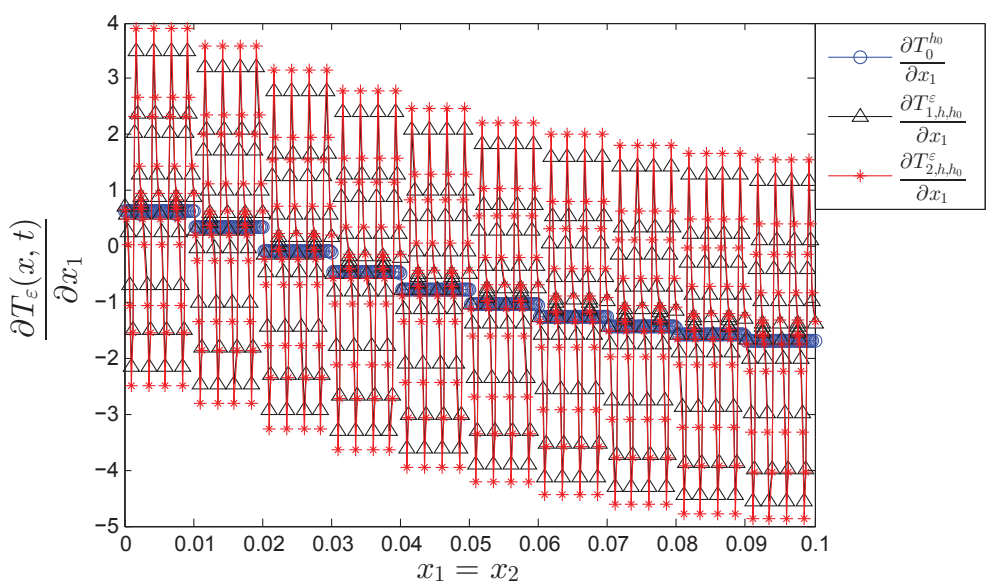

(a)

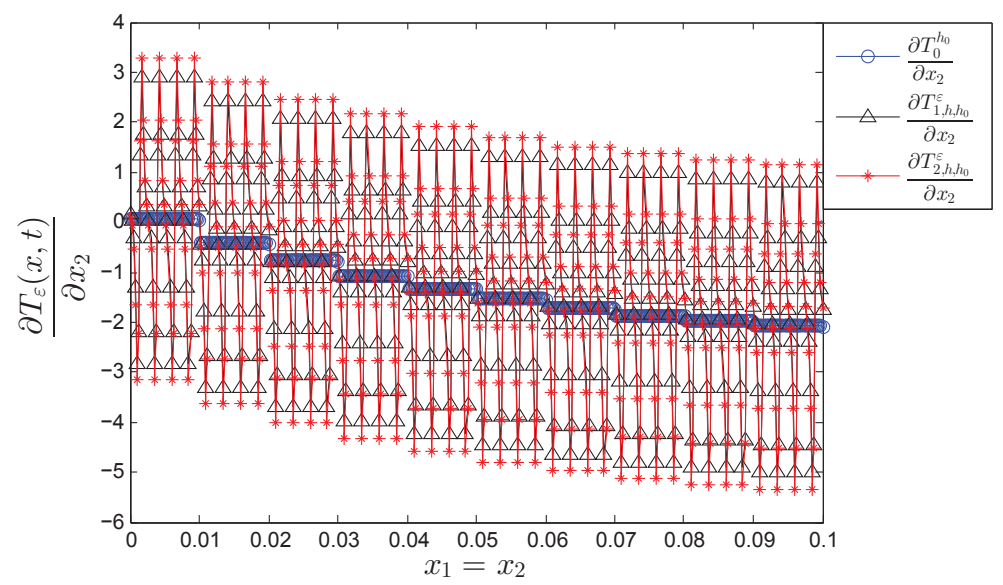

(b)

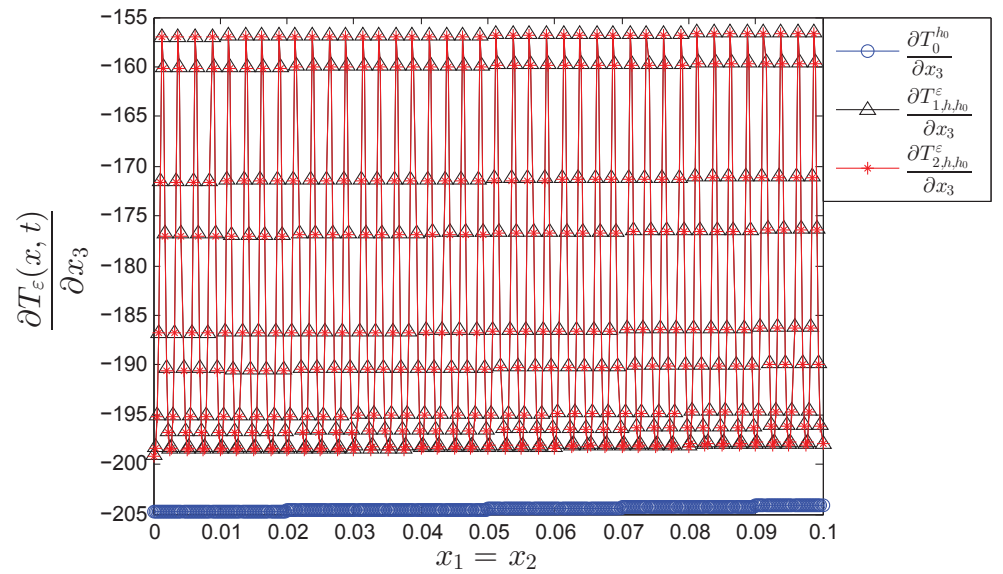

(c)

Figure 12: (a) $\frac{\partial T_{\varepsilon}(x, t)}{\partial x_{1}}$, (b) $\frac{\partial T_{\varepsilon}(x, t)}{\partial x_{2}}$ and (c) $\frac{\partial T_{\varepsilon}(x, t)}{\partial x_{3}}$ along the line of $x_{1}=x_{2}, x_{3}=0.15$ and at time $t=0.2$. 


\section{Conclusions}

In this paper, the heat transfer performance of porous materials with quasi-periodic structures is predicted by the multiscale asymptotic analysis and related numerical algorithms. The second-order multiscale asymptotic expansion formulations for the coupled problems are given, and the convergence results under appropriate assumptions of the homogenized solutions are derived.

Finally, in the numerical examples, it is worth noting that second-order schemes are proposed to effectively acquire local information of the coupled problems, which support the theoretical results of this paper and demonstrate that the multiscale method proposed in this paper is stable and efficient.

\section{Acknowledgments}

This work is supported by the Fundamental Research Funds for the Central Universities, the National Natural Science Foundation of China (11701123) and also supported by China Postdoctoral Science Foundation (2015M580256, 2016T90276).

\section{References}

[1] A. Bensoussan, J. L. Lions and G. Papanicolaou, Asymptotic Analysis for Periodic Structure, North-Holland, Amsterdam, 1978.

[2] O. A. Oleinik, A. S. Shamaev and G. A. Yosifian, Mathematical Problems in Elasticity and Homogenization, North-Holland, Amsterdam, 1992.

[3] V.V. Jikov, S.M. Kozlov and O.A. Oleinik, Homogenization of Differential Operators and Integral Functions, Berlin: Springer, 1994.

[4] I. Temizer and P.Wriggers, Homogenization in finite thermoelasticity, J. Mech. Phys. Solids, 59 (2011), 344-372.

[5] H. Dong, Y. F. Nie, J.Z.Cui and Z.H.Yang, Second-order two-scale computational method for nonlinear dynamic thermo-mechanical problems of composites with cylindrical periodicity, Commun. Comput. Phys., 21(4) (2017), 1173-1206.

[6] H. W. Zhang, D. S. Yang, S. Zhang and Y. G. Zheng, Multiscale nonlinear thermoelastic analysis of heterogeneous multiphase materials with temperature-dependent properties, Finite Elem. Anal. Des., 88 (2014), 97-117.

[7] T. Y. Hou and X. H. Wu, A multiscale finite element method for elliptic problems in composite materials and porous media, J. Comput. Phys., 134 (1997), 169-189.

[8] K. Terada, M. Kurumatani, T. Ushida and N. Kikuchi, A method of two-scale thermomechanical analysis for porous solids with micro-scale heat transfer, Comp. Mech., 46 (2010), 269-285.

[9] F. Su, J. Z. Cui, Z. Xu and Q. L. Dong, A second-order and two-scale computation method for the quasi-periodic structures of composite materials, Finite Elem. Anal. Des., 46 (2010), 320-327. 
[10] F. Han, J. Z. Cui and Y. Yu, The statistical second-order two-scale method for mechanical properties of statistically inhomogeneous materials, Int. J. Numer. Meth. Eng., 84 (2010), 972-988.

[11] S. T. Liu and Y. C. Zhang, Multiscale analysis method for thermal conductivity of porous material with radiation, Multidiscipline Modeling in Mat. and Str., 2(3) (2006), 327-344.

[12] N. S. Bakhvalov, Averaging of the heat-transfer process in periodic media with radiative, Differ. Uraun., 17 (10) (1981), 1765-1773.

[13] G. Allaire and K. El Ganaoui, Homogenization of a conductive and radiative heat transfer problem, Multiscale Model.Sim., 7 (2009), 1148-1170.

[14] Q. Ma and J. Z. Cui, Second-order two-scale analysis method for the heat conductive problem with radiation boundary condition in periodical porous domains, Commun. Comput. Phys., 14(4) (2012), 1027-1057.

[15] Z. Q. Yang, J. Z. Cui, Y.F. Nie and Q. Ma, The second-order two-scale method for heat transfer performances of periodic porous materials with interior surface radiation, CMES: Comp. Model. Eng., 88(5) (2012), 419-442.

[16] Z. Q. Yang, J. Z. Cui and Q. Ma, The second-order two-scale computation for integrated heat transfer problem with conduction,convection and radiation in periodic porous materials, Discret. Contin. Dyn-B, 19(3) (2014), 827-848.

[17] Z.Q. Yang, J. Z. Cui, Y. Sun and J.R. Ge, Multiscale computation for transient heat conduction problem with radiation boundary condition in porous materials, Finite Elem. Anal. Des., 102-103 (2015), 7-18.

[18] Z. Q. Yang, J. Z. Cui, Y. Sun, J. Liang and Z. H. Yang, Multiscale analysis method for thermomechanical performance of periodic porous materials with interior surface radiation, Int. J. Numer. Meth. Eng., 105(53) (2016), 323-350.

[19] Z. Q. Yang, J. Z. Cui, Y. Sun, Y.Z. Liu and Y.C. Xiao, A stochastic multiscale method for thermo-mechanical analysis arising from random porous materials with interior surface radiation, Adv. Eng. Softw., 104 (2017), 12-27.

[20] H.M. Yin, G.H. Paulino and W.G. Buttlar, et al., Micromechanics-based thermoelastic model for functionally graded particulate materials with particle interactions, J. Mech. Phys. Solids, 55 (2007), 132-160.

[21] Z.Q. Yang, J.Z. Cui and Y.Sun, Transient heat conduction problem with radiation boundary condition of statistically inhomogeneous materials by second-order two-scale method, Int. J. Heat Mass Tran., 100 (2016), 362-377.

[22] V.P.W. Shim, L.M. Yang, J.F. Liu and V. S. Lee, Characterisation of the dynamic compressive mechanical properties of cancellous bone from the human cervical spine, Int. J. Impact Eng., 32 (2005), 525-540.

[23] S. Hedayatrasa, T. Q. Bui, C.Z. Zhang and C. W. Lim, Numerical modeling of wave propagation in functionally graded materials using time-domain spectral Chebyshev elements, J. Comput. Phys., 258 (2014), 381-404.

[24] A. Wirth, An extension of spectral methods to quasi-periodic and multiscale problems, J.Comput. Phys., 132(2) (1997), 285-290.

[25] I. V. Andrianov, J. Awrejcewicz and A.A. Diskovsky, Homogenization of quasi-periodic structures, J.Vib. Acoust., 128(4) (2006), 532-534.

[26] L. Cao and J. Cui, Homogenization method for the quasi-periodic structures of composite materials, Math. Numer. Sin., 21 (1999), 331-344.

[27] F. Su, J.Z. Cui, Z. Xu and Q.L. Dong, Multiscale method for the quasi-periodic structures of composite materials, Appl. Math. Comput., 217 (2011), 5847-5852. 
[28] Q. Ma and J. Cui, Second-order two-scale analysis method for the quasi-periodic structure of composite materials under condition of coupled thermo-elasticity, Adv. Mater. Res., 629 (2013), 160-164.

[29] H. Dong, Y.F. Nie, J.Z. Cui, Y.T. Wu and Z.H. Yang, Second-order two-scale analysis and numerical algorithm for the damped wave equations of composite materials with quasiperiodic structures, Appl. Math. Comput., 298 (2017), 201-220.

[30] M. C. Delfour, G. Payre and J. P. ZoleHsio, Approximation of nonlinear problems associated with radiating bodies in space, SIAM J. Numer. Anal., 24 (1987), 1077-1094.

[31] K. El Ganaoui, Homognisation de modles de transferts thermiques et radiatifs:Application au coeur des racteurs A caloporteur gaz. PhD thesis, Ecole Polytechnique, 2006.

[32] F. Su, Z. Xu, Q. L. Dong and H. S. Li, A second-order and two-scale computation method for heat conduction equation with rapidly oscillatory coefficients, Finite Elem. Anal. Des., 47 (2011), 276-280.

[33] J. Z. Cui, The two-scale expression of the solution for the structure with several sub-domains of small periodic configurations, Invited Presentation "Workshop on Scientific Computing 99" June 27-30, 1996, Hong Kong. 